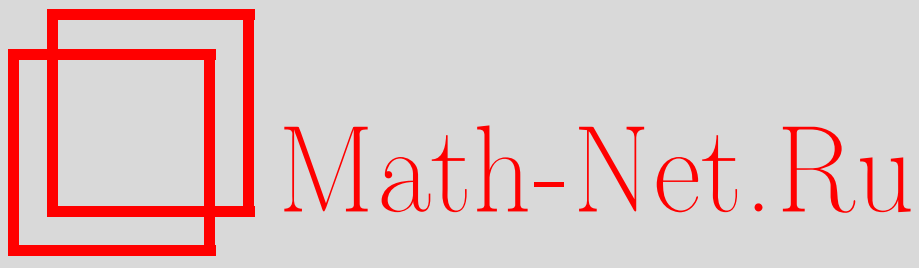

С. В. Асташкин, Системы случайных величин, эквивалентные по распределению системе Радемахера, и $\mathscr{K}$-замкнутая представимость банаховых пар, Матем. сб., 2000, том 191, номер 6, 3-30

DOI: https://doi.org/10.4213/sm481

Использование Общероссийского математического портала Math-Net.Ru подразумевает, что вы прочитали и согласны с пользовательским соглашением

http: //www. mathnet.ru/rus/agreement

Параметры загрузки:

IP : 54.162.85.209

26 апреля 2023 г., 14:42:21 


\title{
С.В. Асташкин
}

\section{Системы случайных величин, эквивалентные по распределению системе Радемахера, и $\mathscr{K}$-замкнутая представимость банаховых пар}

\begin{abstract}
В работе найдены необходимые и достаточные условия того, чтобы из последовательности $\left\{f_{n}\right\}_{n=1}^{\infty}$ случайныт величин (с.в.), определенных на вероятностном пространстве $(\Omega, \Sigma, \mathrm{P})$, можно было выделить подсистему $\left\{\varphi_{i}\right\}_{i=1}^{\infty}$, эквивалентную по распределению системе Радемахера на $[0,1]$. В частности, это всегда возможно, если $\left\{f_{n}\right\}_{n=1}^{\infty}$ - равномерно ограниченная и ортонормированная последовательность. Основную роль в доказательствах играет обнаруженная в работе связь между эквивалентностью по распределению систем с.в. и поведением $L_{p}$-норм соответствующих полиномов.

Приводится приложение полученных результатов к изучению $\mathscr{K}$-замкнутой представимости некоторых банаховых пар.

Библиографияя: 26 названий.
\end{abstract}

\section{Введение}

Системы $\left\{f_{n}\right\}_{n=1}^{\infty}$ и $\left\{g_{n}\right\}_{n=1}^{\infty}$ случайных величин (с.в.), определенных на вероятностных пространствах $(\Omega, \Sigma, \mathrm{P})$ и $\left(\Omega^{\prime}, \Sigma^{\prime}, \mathrm{P}^{\prime}\right)$ соответственно, назовем әквивалентными по распределению (пишем: $\left\{f_{n}\right\} \stackrel{P}{\sim}\left\{g_{n}\right\}$ ), если сушествует $C>0$ такое, что для произвольных $m \in \mathbb{N}, a_{n} \in \mathbb{R}(n=1,2, \ldots, m)$ и $z>0$

$$
\begin{aligned}
C^{-1} \mathrm{P}\left\{\left|\sum_{n=1}^{m} a_{n} f_{n}(\omega)\right|>C z\right\} & \leqslant \mathrm{P}^{\prime}\left\{\left|\sum_{n=1}^{m} a_{n} g_{n}\left(\omega^{\prime}\right)\right|>z\right\} \\
& \leqslant C \mathrm{P}\left\{\left|\sum_{n=1}^{m} a_{n} f_{n}(\omega)\right|>C^{-1} z\right\} .
\end{aligned}
$$

Если выполнена только левая часть этого соотношения, то будем говорить, что система $\left\{f_{n}\right\}_{n=1}^{\infty}$ мажсорируется по распределению системой $\left\{g_{n}\right\}_{n=1}^{\infty}$. Аналогичным образом определяются эти понятия в случае конечных наборов $\left\{f_{n}\right\}_{n=1}^{N}$ и $\left\{g_{n}\right\}_{n=1}^{N}$.

В работе [1] показано, что из всякой равномерно ограниченной ортонормированной в $L_{2}$ последовательности с.в. $\left\{f_{n}\right\}_{n=1}^{\infty}$ можно выделить подпоследовательность $\left\{f_{n_{k}}\right\}_{k=1}^{\infty}$, которая мажорируется по распределению системой Радемахера $\left\{r_{n}\right\}_{n=1}^{\infty}$,

$$
r_{n}(x)=\operatorname{sign} \sin \left(2^{n-1} \pi x\right) \quad(x \in[0,1]) .
$$

Основная цель данной работы - доказать возможность выделения при тех же условиях подпоследовательности, которая не только мажорируется, но и эквивалентна системе $\left\{r_{n}\right\}_{n=1}^{\infty}$. Более того, будут найдены необходимые и достаточные 
условия на систему с.в. такие, чтобы она содержала подсистему, эквивалентную по распределению системе Радемахера. В случае конечных наборов нас будет интересовать плотность их подсистем, обладающих аналогичным свойством.

Проблематика, связанная с выделением “почти независимых" подсистем (т.е. обладающих некоторым свойством, характерньм для систем независимых функций), подробно рассматривается в известной статье В. Ф. Гапошкина “Лакунарные ряды и независимые функции" [2], на которую в дальнейшем мы не раз будем ссылаться. Напомним наиболее важные результаты, относящиеся к интегрируемости суммы ряда, построенного по данной системе функций, а также к его абсолютной сходимости.

Ввиду классического неравенства, доказанного А.Я. Хинчиньгм [3], сумма ряда $\sum_{n=1}^{\infty} a_{n} r_{n}(x)(x \in[0,1])$ принадлежит всем пространствам $L_{p}(p>2)$ при условии, что последовательность коэффициентов $a=\left(a_{n}\right)_{n=1}^{\infty} \in l_{2}$. Для лакунарных тригонометрических рядов аналогичный результат был получен А. Зигмундом [4]. В связи с этим несколько позднее в работах С. Банаха и С. Сидона возникло понятие лакунарной системы порядка $p$, или, короче, $S_{p}$-системы. Последовательность с.в. $\left\{f_{n}\right\}_{n=1}^{\infty}, f_{n} \in L_{p}(p>2)$, назьвается $S_{p}$-системой, если

$$
\left\|\sum_{n=1}^{m} a_{n} f_{n}\right\|_{p} \leqslant K_{p}\left\|\sum_{n=1}^{m} a_{n} f_{n}\right\|_{2}
$$

где константа $K_{p}>0$ не зависит от $m \in \mathbb{N}$ и $a_{n} \in \mathbb{R}(n=1,2, \ldots, m)$. Если $\left\{f_{n}\right\}$ является $S_{p}$-системой при любом $p>2$, то ее называют $S_{\infty}$-системой.

С. Банах доказал, что из всякой ортонормированной последовательности $\left\{f_{n}\right\}$ измеримых функций, определенных на $[0,1]$, такой, что $\lim \sup _{n \rightarrow \infty}\left\|f_{n}\right\|_{p}<\infty$, можно извлечь $S_{p}$-подсистему ([5] или $\left.[6 ; \S 7.2]\right)$. Отсюда, в частности, следует возможность выделения $S_{\infty}$-подсистемы из любой равномерно ограниченной ортонормированной системы функций. Приведем более точный результат, принадлежащий С.Б. Стечкину [2; теорема 1.3.1].

Теорема А. Пусть $\left\{f_{n}\right\}_{n=1}^{\infty}-$ последовательность функиий, измеримых на $[0,1]$, и $p>2$. Для того чтобь из $\left\{f_{n}\right\}_{n=1}^{\infty}$ можсно било извлечь $S_{p}$-подсистему, необходимо и достаточно существования подпоследовательности $\left\{f_{n_{k}}\right\}$, для которой выполнены условия:

1) $\left\|f_{n_{k}}\right\|_{p} \leqslant D(k=1,2, \ldots)$;

2) $f_{n_{k}} \rightarrow 0$ слабо в $L_{2}$.

Перейдем теперь к краткому обсуждению другого свойства лакунарных рядов - абсолютной сходимости. Для тригонометрических рядов хорошо известна классическая теорема Сидона об абсолютной сходимости лакунарного (в смысле Адамара) ряда Фурье ограниченной функции. А. Зигмунд доказал локальный вариант этой теоремы [7]. Позднее аналогичные результаты были получены для системы Радемахера и некоторых других конкретных лакунарных систем функций [8]. Как естественное обобщение этих результатов возникли следующие понятия. Последовательность с.в. $\left\{f_{n}\right\}_{n=1}^{\infty}$ называется системой Сидона, если

$$
\sum_{n=1}^{m}\left|a_{n}\right| \leqslant C\left\|\sum_{n=1}^{m} a_{n} f_{n}\right\|_{\infty},
$$


где константа $C>0$ не зависит от $m \in \mathbb{N}$ и $a_{n} \in \mathbb{R}(n=1,2, \ldots, m)$.

Систему $\left\{f_{n}\right\}_{n=1}^{\infty}$ функций, измеримых на $[0,1]$, называют системой Cидона-Зигмунда, если сушествует множество $E \subset[0,1]$ с мерой Лебега $|E|>0$ такое, что для всякого промежутка $I,|I \cap E|>0$, из условия $\sum_{n=1}^{\infty} a_{n} f_{n}(x) \in L_{\infty}(I)$ следует: $\left(a_{n}\right)_{n=1}^{\infty} \in l_{1}$. Если в этом определении можно взять $E=[0,1]$, то говорят, что $\left\{f_{n}\right\}_{n=1}^{\infty}-$ система Сидона-Зигмунда в узком смысле.

Широкие достаточные условия, обеспечивающие возможность выделения подсистем Сидона и Сидона-Зигмунда, были получены В. Ф. Гапошкиным [2; теоремы 1.4.1 и 1.4.2].

ТеОремА В. Пусть система $\left\{f_{n}\right\}_{n=1}^{\infty}$ функиий, измеримых на $[0,1]$, обладает следующими свойствами:

1) $\left\|f_{n}\right\|_{2}=1(n=1,2, \ldots)$;

2) $\left|f_{n}(x)\right| \leqslant D(n=1,2, \ldots ; x \in[0,1])$;

3) существует подпоследовательность $\left\{f_{n_{k}}\right\} \subset\left\{f_{n}\right\}$ такая, ито $f_{n_{k}} \rightarrow 0$ слабо в $L_{2}$.

Тогда из $\left\{f_{n}\right\}_{n=1}^{\infty}$ можсно выделить подсистему Сидона.

ТЕОРема В'. а) При выполнении условий теоремы В из системы $\left\{f_{n}\right\}_{n=1}^{\infty}$ можно выделить подсистему Сидона-Зигмунда.

b) Если дополнительно подпоследовательность из условия 3) теоремы В удовлетворяет условию

$$
\liminf _{k \rightarrow \infty} \int_{F} f_{n_{k}}^{2}(x) d x>0 \quad \text { для любого } F \subset[0,1], \quad|F|>0
$$

то из системы $\left\{f_{n}\right\}_{n=1}^{\infty}$ моэсно выделить подсистему Сидона-Зигмунда в узком смисле.

Из приведенных выше определений и свойств системы Радемахера следует, что если $\left\{f_{n}\right\} \stackrel{\text { P }}{\sim}\left\{r_{n}\right\}$, то:

1) $\left\{f_{n}\right\}-S_{\infty}$-система, причем константа $K_{p}$ из неравенства $(0.1)$ имеет такой же порядок роста по $p$ при $p \rightarrow \infty$, как и для системы Радемахера, т.е. $K_{p} \asymp \sqrt{p}$

2) $\left\{f_{n}\right\}$ - система Сидона.

Тем самым утверждения, доказанные ниже в $\S 3$, обеспечивают "хорошие" свойства выделенной подсистемы в обоих отношениях, усиливая и дополняя приведенные результаты.

Наряду с вопросами выделения бесконечных подпоследовательностей, обладающих свойствами систем независимых функций, представляют интерес также задачи иного рода, речь в которых идет о конечных наборах с.в. Предположим, что $\left\{f_{n}\right\}_{n=1}^{N}-$ набор с.в. Требуется найти как можно большее $s=s(N)$ со следующим свойством: существует набор $\left\{f_{n_{i}}\right\}_{i=1}^{s} \subset\left\{f_{n}\right\}_{n=1}^{N}$, удовлетворяюший некоторому условию лакунарности с константой, не зависяшей от $N$. Приведем лишш один результат о плотности конечных подсистем Сидона, доказанный Б.С. Кашиным (см. [9] или $[10 ; \S 8.4$, теорема 9]).

Теорема С. Пусть $\left\{f_{n}\right\}_{n=1}^{N}-$ ортонормированный набор функиий, определенных на $[0,1],\left|f_{n}(x)\right| \leqslant D(n=1, \ldots, N ; x \in[0,1])$. Тогда существует набор 
$\left\{f_{n_{i}}\right\}_{i=1}^{s} \subset\left\{f_{n}\right\}_{n=1}^{N}\left(1 \leqslant n_{1}<\cdots<n_{s} \leqslant N\right)$ maкой, что $s \geqslant \max \left\{\left[\frac{1}{6} \log _{2} N\right] ; 1\right\}$ и для любих $a_{i} \in \mathbb{R}(i=1, \ldots, s)$ выполнено:

$$
D^{-1}\left\|\sum_{i=1}^{s} a_{i} f_{n_{i}}\right\|_{\infty} \leqslant \sum_{i=1}^{s}\left|a_{i}\right| \leqslant 4 D\left\|\sum_{i=1}^{s} a_{i} f_{n_{i}}\right\|_{\infty} .
$$

Здесь при тех же предположениях будет доказано утверждение о выделении подсистемы $\left\{f_{n_{i}}\right\}_{i=1}^{s}$ такой же плотности, но удовлетворяющей более сильному условию: она эквивалентна системе $\left\{r_{i}\right\}_{i=1}^{s}$ с константой, зависящей только от $D$.

Работа состоит из пяти параграфов. В $\S 1$ собраны используемые в дальнейшем понятия теории интерполяции операторов (подробнее см. в [11]). Для полноты изложения здесь же приведено доказательство одной формулы для $\mathscr{K}$-функционала, построенного по паре $\left(l_{1}, l_{2}\right)$, из работы [12]. В $\S 2$ доказан критерий эквивалентности по распределению произвольной последовательности с.в. системе Радемахера, получены следствия в некоторых конкретных случаях. Основные результаты статьи - о выделении подсистем, эквивалентных по распределению системе Радемахера, - составляют содержание $\S 3$. Следуюший параграф̆ посвяшен рассмотрению аналогичных вопросов в случае конечных наборов функций. И наконец, в $\S 5$ полученные результаты применяются к изучению $\mathscr{K}$-замкнутой представимости банаховых пар.

В заключение приведем обозначения. Выражение вида $F_{1} \asymp F_{2}$ в дальнейшем означает, что для некоторого $C>0 C^{-1} F_{1} \leqslant F_{2} \leqslant C F_{1}$, причем константа $C$, как правило, не зависит от всех или части аргументов $F_{1}$ и $F_{2}$. Если $1 \leqslant p \leqslant \infty$, $f=f(\omega)-$ с.в. на вероятностном пространстве $(\Omega, \Sigma, \mathrm{P})$, то

$$
\|f\|_{p}=\left(\mathrm{E}|f|^{p}\right)^{1 / p}=\left\{\int_{\Omega}|f(\omega)|^{p} d \mathrm{P}(\omega)\right\}^{1 / p}
$$

если же $a=\left(a_{n}\right)_{n=1}^{\infty}-$ числовая последовательность, то

$$
\|a\|_{p}=\left\{\sum_{n=1}^{\infty}\left|a_{n}\right|^{p}\right\}^{1 / p}
$$

(с естественной модификацией формул при $p=\infty$ ). Как обычно, пространство $L_{p}$ состоит из всех с.в. $f$, для которых $\|f\|_{p}<\infty$, а $l_{p}$ - из всех последовательностей $a=\left(a_{n}\right)_{n=1}^{\infty}$ таких, что $\|a\|_{p}<\infty$.

Через $|E|$ будет обозначаться мера Лебега множества $E \subset[0,1]$.

\section{§1. $\mathscr{K}$-функционал Петре и вещественный метод интерполяции}

Пусть $\left(X_{0}, X_{1}\right)$ - банахова пара, $x \in X_{0}+X_{1}, t>0$. Определим $\mathscr{K}$-функционал Петре

$$
\mathscr{K}\left(t, x ; X_{0}, X_{1}\right)=\inf \left\{\left\|x_{0}\right\|_{X_{0}}+t\left\|x_{1}\right\|_{X_{1}}: x=x_{0}+x_{1}, x_{0} \in X_{0}, x_{1} \in X_{1}\right\}
$$

играющий важную роль в теории интерполяции операторов.

Нетрудно показать, что при фиксированном $x \in X_{0}+X_{1} \mathscr{K}$-функционал - непрерывная возрастающая вогнутая функция относительно переменной $t[11 ; \S 3.1]$. 
В дальнейшем нам будет нужен лишь функционал $\mathscr{K}_{1,2}(t, a)=\mathscr{K}\left(t, a ; l_{1}, l_{2}\right)$, построенный по банаховой паре $\left(l_{1}, l_{2}\right)$, две аппроксимации которого составляют содержание следующих лемм. Первая из них, принадлежащая С. Монтгомери-Смиту, будет сушественно использована в доказательстве теоремы 4. Поэтому для полноты изложения мы приведем ее доказательство (см. [12]).

Для произвольных $a=\left(a_{n}\right)_{n=1}^{\infty}$ и $t \in \mathbb{N}$ определим норму

$$
\|a\|_{Q(t)}=\sup \left\{\sum_{j=1}^{t}\left(\sum_{n \in A_{j}} a_{n}^{2}\right)^{1 / 2}\right\},
$$

где верхняя грань берется по всем разбиениям $\left\{A_{j}\right\}_{j=1}^{t}$ множества натуральных чисел $\mathbb{N}$.

Лемма 1. Если $a=\left(a_{n}\right)_{n=1}^{\infty} \in l_{2} u t^{2} \in \mathbb{N}, m o$

$$
\|a\|_{Q\left(t^{2}\right)} \leqslant \mathscr{K}_{1,2}(t, a) \leqslant \sqrt{2}\|a\|_{Q\left(t^{2}\right)} .
$$

ДокАЗАТЕЛЬСТво. Из определения $Q(t)$ следует, что

$$
\|a\|_{Q\left(t^{2}\right)} \leqslant\|a\|_{1} \quad \text { и }\|a\|_{Q\left(t^{2}\right)} \leqslant t\|a\|_{2} .
$$

Поэтому

$$
\begin{aligned}
\mathscr{K}_{1,2}(t, a) & =\inf \left\{\|b\|_{1}+t\|c\|_{2}: b+c=a, b \in l_{1}, c \in l_{2}\right\} \\
& \geqslant \inf \left\{\|b\|_{Q\left(t^{2}\right)}+\|c\|_{Q\left(t^{2}\right)}: b+c=a, b \in l_{1}, c \in l_{2}\right\} \geqslant\|a\|_{Q\left(t^{2}\right)}
\end{aligned}
$$

и левая часть (1.2) доказана.

Для доказательства правой части (1.2) прежде всего заметим, что

$$
\mathscr{K}_{1,2}(t, a)=\sup \left\{\sum_{n=1}^{\infty} a_{n} b_{n}: b=\left(b_{n}\right)_{n=1}^{\infty} \in l_{2}, \mathscr{J}_{\infty, 2}\left(t^{-1}, b\right) \leqslant 1\right\},
$$

где $\mathscr{J}_{\infty, 2}(s, b)=\max \left\{\|b\|_{\infty} ; s\|b\|_{2}\right\}$ (см. $[11 ; \S 2.7]$ ).

Следовательно, для всякого $\delta>0$ найдется последовательность $b \in l_{2}$ такая, что

$$
(1-\delta) \mathscr{K}_{1,2}(t, a) \leqslant \sum_{n=1}^{\infty} a_{n} b_{n} \text { и } \quad \mathscr{J}_{\infty, 2}\left(t^{-1}, b\right)=1 .
$$

Выберем $n_{0}, n_{1}, n_{2}, \ldots, n_{t^{2}} \in\{0,1, \ldots, \infty\}$ по индукции следующим образом: если $0=n_{0}<n_{1}<\cdots<n_{m}$ уже найдены, то

$$
n_{m+1}=1+\sup \left\{k: \sum_{n=n_{m}+1}^{k} b_{n}^{2} \leqslant 1\right\} .
$$

Так как $\|b\|_{\infty} \leqslant 1$, то $\sum_{n=n_{m}+1}^{n_{m+1}} b_{n}^{2} \leqslant 2$. Ввиду того, что $\|b\|_{2} \leqslant t, n_{t^{2}}=\infty$.

Следовательно,

$$
\begin{aligned}
(1-\delta) \mathscr{K}_{1,2}(t, a) & \leqslant \sum_{n=1}^{\infty} a_{n} b_{n} \\
& \leqslant \sum_{m=1}^{t^{2}}\left(\sum_{n=n_{m-1}+1}^{n_{m}} b_{n}^{2}\right)^{1 / 2}\left(\sum_{n=n_{m-1}+1}^{n_{m}} a_{n}^{2}\right)^{1 / 2} \leqslant \sqrt{2}\|a\|_{Q\left(t^{2}\right)} .
\end{aligned}
$$

Так как последнее справедливо для всех $\delta>0$, то лемма доказана.

Другое соотношение - формула Т. Хольмстедта - хорошо известна (см. [13] или $[11 ; \S 5.7])$. 
Лемма 2. Существует константа $\alpha>0$, не зависящая от $a=\left(a_{n}\right)_{n=1}^{\infty} \in l_{2}$ $u t>0$, такая, что

$\alpha^{-1}\left\{\sum_{i=1}^{\left[t^{2}\right]} a_{i}^{*}+t\left[\sum_{i=\left[t^{2}\right]+1}^{\infty}\left(a_{i}^{*}\right)^{2}\right]^{1 / 2}\right\} \leqslant \mathscr{K}_{1,2}(t, a) \leqslant\left\{\sum_{i=1}^{\left[t^{2}\right]} a_{i}^{*}+t\left[\sum_{i=\left[t^{2}\right]+1}^{\infty}\left(a_{i}^{*}\right)^{2}\right]^{1 / 2}\right\}$,

әде $\left(a_{i}^{*}\right)_{i=1}^{\infty}-$ убьвающая перестановка последовательности $\left(\left|a_{n}\right|\right)_{n=1}^{\infty}, a[z]-$ иелая часть числа $z$.

СлЕДСТВИЕ 1. Функиия $\kappa(t, a)=\mathscr{K}_{1,2}(\sqrt{t}, a)$ непрерывна и возрастает при $t \geqslant 0$. Для любой последовательности $a=\left(a_{n}\right)_{n=1}^{\infty} \in l_{2}$ :

1) $\lim _{t \rightarrow 0+} \kappa(t, a)=0$;

2) $\alpha^{-1}\|a\|_{1} \leqslant \lim _{t \rightarrow+\infty} \kappa(t, a) \leqslant\|a\|_{1}$.

В заключение параграфа напомним определение вешественного метода интерполяции. Для произвольной банаховой пары $\left(X_{0}, X_{1}\right), 0<\theta<1,1 \leqslant q \leqslant \infty$ определим норму

$$
\|x\|_{\theta, q}=\left\{\sum_{n=-\infty}^{\infty}\left[2^{-n \theta} \mathscr{K}\left(2^{n}, x ; X_{0}, X_{1}\right)\right]^{q}\right\}^{1 / q}, \quad x \in X_{0}+X_{1}
$$

(с естественной модификацией при $q=\infty)$. Пространства $\left(X_{0}, X_{1}\right)_{\theta, q}=\{x \in$ $\left.X_{0}+X_{1}:\|x\|_{\theta, q}<\infty\right\}$ интерполяционны относительно пары $\left(X_{0}, X_{1}\right)$ (т.е. любой линейньй оператор, ограниченный в $X_{0}$ и в $X_{1}$, ограничен в $\left.\left(X_{0}, X_{1}\right)_{\theta, q}\right)$ и называются пространствами вещественного метода интерполяиии.

\section{§ 2. Системы с.в., эквивалентные по распределению системе Радемахера}

ТЕОРема 1. Пусть $\left\{f_{n}\right\}_{n=1}^{\infty}-$ последовательность с.в., определенных на вероятностном пространстве $(\Omega, \Sigma, \mathrm{P})$. Следующие условия әквивалентнь:

1) $\left\{f_{n}\right\} \stackrel{\mathrm{P}}{\sim}\left\{r_{n}\right\}\left(\left\{r_{n}\right\}_{n=1}^{\infty}-\right.$ система Радемахера на $\left.[0,1]\right)$;

2) с константой, не зависящей от $t \in[1, \infty), m \in \mathbb{N}$ и $a_{n} \in \mathbb{R}$ $(n=1, \ldots, m)$,

$$
\left\|\sum_{n=1}^{m} a_{n} f_{n}\right\|_{t} \asymp\left\|\sum_{n=1}^{m} a_{n} r_{n}\right\|_{t}
$$

3) с константой, не зависящей от $t \in[1, \infty)$ и $a=\left(a_{n}\right)_{n=1}^{\infty} \in l_{2}$,

$$
\left\|\sum_{n=1}^{\infty} a_{n} f_{n}\right\|_{t} \asymp \mathscr{K}_{1,2}(\sqrt{t}, a)
$$


ДокАЗАТЕЛЬСтво. В работе [14] доказано, что с константой, не зависящей от $t \in[1, \infty)$ и $a=\left(a_{n}\right)_{n=1}^{\infty} \in l_{2}$,

$$
\left\|\sum_{n=1}^{\infty} a_{n} r_{n}\right\|_{t} \asymp \mathscr{K}_{1,2}(\sqrt{t}, a)
$$

(неявно это соотношение содержится уже в работе [12]). Поэтому эквивалентность $2) \Leftrightarrow 3)$ очевидна. Так как импликация 1) $\Rightarrow 2$ ) является следствием определения эквивалентности систем по распределению, то остается доказать лишь импликацию 3$) \Rightarrow 1$ ).

Зафиксируем $a=\left(a_{n}\right)_{n=1}^{m}, m \in \mathbb{N}$ (не все $a_{n}$ равны нулю), и покажем, что распределение модуля с.в.

$$
f(\omega)=\sum_{n=1}^{m} a_{n} f_{n}(\omega) \quad(\omega \in \Omega)
$$

определяется поведением ее моментов $\|f\|_{t}$, а значит, по условию функцией $\kappa(t, a)=$ $\mathscr{K}_{1,2}(\sqrt{t}, a)(t \geqslant 1)$.

Обозначив через $\beta \geqslant 1$ константу эквивалентности (2.1), ввиду неравенства Пэли-Зигмунда $[15 ; \S 1.6]$ и вогнутости $\mathscr{K}$-функционала для $t \geqslant 1$ получим

$$
\begin{aligned}
\mathrm{P}\{|f(\omega)| & \left.\geqslant(2 \beta)^{-1} \kappa(t, a)\right\} \geqslant \mathrm{P}\left\{|f(\omega)|^{t} \geqslant 2^{-t}\|f\|_{t}^{t}\right\} \\
& \geqslant\left(1-2^{-t}\right)^{2} \frac{\|f\|_{t}^{2 t}}{\|f\|_{2 t}^{2 t}} \geqslant \beta^{-4 t}\left(1-2^{-t}\right)^{2}\left\{\frac{\kappa(t, a)}{\kappa(2 t, a)}\right\}^{2 t} \geqslant(2 \beta)^{-4 t} .
\end{aligned}
$$

Так как функция $\kappa(t, a)$ возрастает по $t$, то отсюда

$$
\mathrm{P}\left\{|f(\omega)| \geqslant(2 \beta)^{-1} \kappa(t, a)\right\} \geqslant(2 \beta)^{-4 t-4}
$$

уже для всех $t>0$.

Наоборот, из неравенства Чебьшёва и условия (2.1) получаем

$$
\mathrm{P}\{|f(\omega)| \geqslant \lambda \kappa(t, a)\} \leqslant \lambda^{-t}\left[\frac{\|f\|_{t}}{\kappa(t, a)}\right]^{t} \leqslant\left(\frac{\beta}{\lambda}\right)^{t}
$$

для произвольных $t \geqslant 1$ и $\lambda>0$. В частности, если $\lambda=\beta e$, то

$$
\mathrm{P}\{|f(\omega)| \geqslant \beta e \kappa(t, a)\} \leqslant e^{-t} \quad(t \geqslant 1)
$$

или

$$
\mathrm{P}\{|f(\omega)| \geqslant \beta e \kappa(t, a)\} \leqslant e^{1-t} \quad(t>0) .
$$

Следуя [14], определим функционалы

$$
F(s)=\sup \{t>0: \kappa(t, a) \leqslant s\}, \quad G(s)=\inf \{t>0: \kappa(t, a) \geqslant s\} .
$$

Если $0<z<(4 \alpha \beta)^{-1}\|a\|_{1}$, то по следствию 1 найдется $t>0$ такое, что $\kappa(t, a) \geqslant 4 z \beta$. Тогда из (2.3) следует, что

$$
\mathrm{P}\{|f(\omega)|>z\} \geqslant \mathrm{P}\left\{|f(\omega)| \geqslant(2 \beta)^{-1} \kappa(t, a)\right\} \geqslant(2 \beta)^{-4 t-4} .
$$


Значит, по определению функционала $G$

$$
\mathrm{P}\{|f(\omega)|>z\} \geqslant(2 \beta)^{-4 G(4 \beta z)-4},
$$

откуда

$$
\mathrm{P}\{|f(\omega)|>z\} \geqslant C_{1}^{-1} e^{-C_{2} G(4 \beta z)} \quad\left(z<(4 \alpha \beta)^{-1}\|a\|_{1}\right),
$$

где $C_{1}=(2 \beta)^{4}, C_{2}=4 \ln (2 \beta)$.

Покажем, что для $C_{3}=4 \sqrt{2} C_{2} \beta$ выполнено

$$
C_{2} G(4 \beta z) \leqslant F\left(C_{3} z\right) \quad(z>0) .
$$

Действительно, ввиду вогнутости $\mathscr{K}$-функционала и определения $G$

$$
\kappa\left(C_{2} G(4 \beta z), a\right) \leqslant \sqrt{2} C_{2} \kappa\left(2^{-1} G(4 \beta z), a\right) \leqslant 4 \sqrt{2} C_{2} \beta z=C_{3} z
$$

и осталось воспользоваться определением функционала $F$. Таким образом, $(2.5)$ можно переписать в виде

$$
\mathrm{P}\{|f(\omega)|>z\} \geqslant C_{1}^{-1} e^{-F\left(C_{3} z\right)} \quad\left(z<(4 \alpha \beta)^{-1}\|a\|_{1}\right) .
$$

Заметим, что из (2.1) следует

$$
\left\|f_{n}\right\|_{\infty}=\lim _{t \rightarrow+\infty}\left\|f_{n}\right\|_{t} \leqslant \beta
$$

и поэтому $\|f\|_{\infty} \leqslant \beta\|a\|_{1}$. Таким образом,

$$
\mathrm{P}\{|f(\omega)|>z\}=0 \quad\left(z \geqslant \beta\|a\|_{1}\right) .
$$

Далее, по следствию 1 можно найти $t^{\prime}>0$ такое, что $0<\kappa\left(t^{\prime}, a\right) \leqslant(2 \beta e)^{-1} z$. Тогда из (2.4) получаем

$$
\mathrm{P}\{|f(\omega)|>z\} \leqslant \mathrm{P}\left\{|f(\omega)| \geqslant \beta e \kappa\left(t^{\prime}, a\right)\right\} \leqslant e^{1-t^{\prime}},
$$

и значит, по определению $F$

$$
\mathrm{P}\{|f(\omega)|>z\} \leqslant e^{1-F\left(C_{4}^{-1} z\right)} \quad(z>0),
$$

где $C_{4}=2 \beta e$.

Ввиду (2.2) соотношения, аналогичные (2.6)-(2.8), справедливы и для системы Радемахера. А именно, если $\beta^{\prime} \geqslant 1$ - константа эквивалентности (2.2), то для функции $r(x)=\sum_{n=1}^{m} a_{n} r_{n}(x)$ выполнено

$$
\begin{gathered}
|\{|r(x)|>z\}| \geqslant\left(C_{1}^{\prime}\right)^{-1} e^{-F\left(C_{3}^{\prime} z\right)} \quad\left(z<\left(4 \alpha \beta^{\prime}\right)^{-1}\|a\|_{1}\right), \\
|\{|r(x)|>z\}|=0 \quad\left(z \geqslant\|a\|_{1}\right) \\
|\{|r(x)|>z\}| \leqslant e^{1-F\left(\left(C_{4}^{\prime}\right)^{-1} z\right)} \quad(z>0) .
\end{gathered}
$$

Пусть

$$
A=\max \left(C_{1} e ; C_{3} C_{4}^{\prime} ; C_{1}^{\prime} e ; C_{3}^{\prime} C_{4} ; 4 \alpha \beta \beta^{\prime}\right) .
$$


Заметим, что ввиду универсальности констант $\alpha$ и $\beta^{\prime}$ зависимость константы $A$ от системы $\left\{f_{n}\right\}_{n=1}^{\infty}$ исчерпьвается лишь ее зависимостью от $\beta$, константы эквивалентности $(2.1)$.

Если $z<(4 \alpha \beta)^{-1}\|a\|_{1}$, то ввиду соотношений $\left(2.8^{\prime}\right)$ и (2.6), а также выбора константы $A$

$$
\begin{aligned}
|\{|r(x)|>A z\}| & \leqslant e^{1-F\left(\left(C_{4}^{\prime}\right)^{-1} A z\right)} \leqslant e^{1-F\left(C_{3} z\right)} \\
& \leqslant C_{1} e \mathrm{P}\{|f(\omega)|>z\} \leqslant A \mathrm{P}\{|f(\omega)|>z\} .
\end{aligned}
$$

Если же $z \geqslant(4 \alpha \beta)^{-1}\|a\|_{1}$, то из (2.9) следует $A z \geqslant\|a\|_{1}$, и поэтому ввиду $\left(2.7^{\prime}\right)$ $|\{|r(x)|>A z\}|=0$. Таким образом, неравенство (2.10) в этом случае также вьполнено.

Наоборот, если $z / A<\left(4 \alpha \beta^{\prime}\right)^{-1}\|a\|_{1}$, то из неравенств $(2.8),\left(2.6^{\prime}\right)$ и выбора $A$ следует, что

$$
\begin{aligned}
\mathrm{P}\{|f(\omega)|>z\} & \leqslant e^{1-F\left(C_{4}^{-1} z\right)} \leqslant e^{1-F\left(C_{3}^{\prime}(z / A)\right)} \\
& \leqslant C_{1}^{\prime} e\left|\left\{|r(x)|>\frac{z}{A}\right\}\right| \leqslant A\left|\left\{|r(x)|>\frac{z}{A}\right\}\right| .
\end{aligned}
$$

В случае, когда $z / A \geqslant\left(4 \alpha \beta^{\prime}\right)^{-1}\|a\|_{1}$, из (2.9) получаем: $z \geqslant \beta\|a\|_{1}$. Значит, ввиду (2.7) $\mathrm{P}\{|f(\omega)|>z\}=0$ и неравенство (2.11) снова выполнено.

Неравенства $(2.10)$ и (2.11) означают, что $\left\{f_{n}\right\} \stackrel{P}{\sim}\left\{r_{n}\right\}$ и теорема 1 доказана.

ЗАмечание 1. Аналогичное утверждение справедливо, разумеется, и для конечных наборов с.в. При этом доказательство показывает, что константы эквивалентностей из условий 1)-3) теоремы 1 зависят лишш друг от друга. Так, например, если соотношение (2.1) вьполнено для наборов $\left\{f_{n}\right\}_{n=1}^{N}$ с константой, не зависящей от $N=1,2, \ldots$, то $\left\{f_{n}\right\}_{n=1}^{N} \stackrel{P}{\sim}\left\{r_{n}\right\}_{n=1}^{N}$ с константой, также не зависящей от $N=1,2, \ldots$.

Применим доказанную теорему к двум более конкретным случаям.

Напомним, что система с.в. $\left\{f_{n}\right\}_{n=1}^{\infty}$ называется мультипликативной, если для произвольных попарно различных $n_{1}, n_{2}, \ldots, n_{k}(k \in \mathbb{N})$ выполнено

$$
\mathrm{E}\left(f_{n_{1}} f_{n_{2}} \cdots f_{n_{k}}\right)=0 .
$$

Если дополнительно

$$
\mathrm{E}\left(f_{n_{1}} f_{n_{2}} \cdots f_{n_{k}} f_{n}^{2}\right)=0
$$

для любых попарно различных $n_{1}, n_{2}, \ldots, n_{k}$ и $n \neq n_{s}(s=1,2, \ldots, k)$, то систему $\left\{f_{n}\right\}$ будем назьвать сильно мультипликативной. В случае конечных наборов определения совершенно аналогичны.

Приведем наиболее важные примеры таких систем [16].

ПримеР 1 . Пусть $f_{n}(x)=\sin \left(2 \pi k_{n} x\right)(x \in[0,1])$. Если $k_{n+1} / k_{n} \geqslant 2$, то это мультипликативная, а если $k_{n+1} / k_{n} \geqslant 3,-$ сильно мультипликативная система.

ПримеР 2. Последовательность $\left\{f_{n}\right\}_{n=1}^{\infty}$ независимых с.в. таких, что $f_{n} \in L_{2}$ и $\mathrm{E}\left(f_{n}\right)=0(n=1,2, \ldots),-$ сильно мультипликативная система.

С. Квапень и Й. Якубовский доказали следующее утверждение [16]. 
Tеорема D. Пусть $\left\{\varphi_{n}\right\}_{n=1}^{N}-$ мультипликативная, $а\left\{\psi_{n}\right\}_{n=1}^{N}-$ сильно мультипликативная системы с.в. Если для любого $n=1, \ldots, N$

$$
\left\|\psi_{n}\right\|_{\infty}\left\|\varphi_{n}\right\|_{\infty} \leqslant \mathrm{E}\left(\psi_{n}^{2}\right)
$$

то существуют вероятностное пространство $\left(\Omega^{\prime}, \Sigma^{\prime}, \mathrm{P}^{\prime}\right)$, -подалгебра $\Sigma_{0}$ $\sigma$-алгебры $\Sigma^{\prime}$ и определенный на $\left(\Omega^{\prime}, \Sigma^{\prime}, \mathrm{P}^{\prime}\right)$ случайный вектор $\left(\psi_{1}^{\prime}, \psi_{2}^{\prime}, \ldots, \psi_{N}^{\prime}\right)$ такой, что он равнораспределен с вектором $\left(\psi_{1}, \psi_{2}, \ldots, \psi_{N}\right)$, а вектор $\left(\varphi_{1}, \varphi_{2}, \ldots, \varphi_{N}\right)$ равнораспределен с условным математическим ожиданием $\mathrm{E}\left(\left(\psi_{1}^{\prime}, \psi_{2}^{\prime}, \ldots, \psi_{N}^{\prime}\right) \mid \Sigma_{0}\right)$.

$B$ частности, для произвольной въппуклой функции $H: \mathbb{R}^{n} \rightarrow \mathbb{R}$

$$
\mathrm{E}\left(H\left(\varphi_{1}, \ldots, \varphi_{N}\right)\right) \leqslant \mathrm{E}\left(H\left(\psi_{1}, \ldots, \psi_{N}\right)\right) .
$$

Из теорем D и 1 получаем следующее утверждение.

ТЕОРема 2. Пусть $\left\{f_{n}\right\}_{n=1}^{\infty}-$ сильно мультипликативная система с.в. такая, что $\left|f_{n}(\omega)\right| \leqslant D(n=1,2, \ldots ; \omega \in \Omega) u d=\inf _{n=1,2, \ldots} \mathrm{E}\left(f_{n}^{2}\right)>0$. Тогда $\left\{f_{n}\right\} \stackrel{\mathrm{P}}{\sim}\left\{r_{n}\right\}$, причем константа этой эквивалентности зависит только от $D u d$.

ДокАЗАТЕЛЬство. Условие (2.12) теоремы D выполнено для систем $\varphi_{n}=f_{n} / D$ и $\psi_{n}=r_{n}$. Поэтому при всех $t \geqslant 1, m \in \mathbb{N}$ и $a_{n} \in \mathbb{R}(n=1,2, \ldots, m)$

$$
\left\|\sum_{n=1}^{m} a_{n} f_{n}\right\|_{t} \leqslant D\left\|\sum_{n=1}^{m} a_{n} r_{n}\right\|_{t} .
$$

Наоборот, если $\varphi_{n}=(d / D) r_{n}, \psi_{n}=f_{n}$, то (2.12) опять выполнено и, значит,

$$
\left\|\sum_{n=1}^{m} a_{n} r_{n}\right\|_{t} \leqslant \frac{D}{d}\left\|\sum_{n=1}^{m} a_{n} f_{n}\right\|_{t} .
$$

Для завершения доказательства осталось применить теорему 1.

СлЕДСТВИЕ 2. Всякая последовательность независимых с.в. $\left\{f_{n}\right\}_{n=1}^{\infty}$ такая, что $f_{n} \in L_{2}, \mathrm{E}\left(f_{n}\right)=0,\left|f_{n}(\omega)\right| \leqslant D(n=1,2, \ldots ; \omega \in \Omega) u d=$ $\inf _{n=1,2, \ldots} \mathrm{E}\left(f_{n}^{2}\right)>0$, әквивалентна по распределению системе Радемахера.

Рассмотрим теперь иную ситуацию. Пусть $G$ - компактная абелева группа, $\Gamma$ группа характеров, определенных на ней, $\mu$ - мера Хаара на $G$. В соответствии с ранее приведенным определением множество $F \subset \Gamma$ называют множеством Cидона, если при некотором $C=C(F)$

$$
\sum_{\gamma \in \Gamma}|\widehat{f}(\gamma)| \leqslant C\|f\|_{\infty}
$$

для всякой $f \in C(G)$ такой, что

$$
\widehat{f}(\gamma)=\int_{G} f \bar{\gamma} d \mu=0 \quad(\gamma \notin F) .
$$

Ж. Пизье [17] доказал следуюшее утверждение (мы приведем формулировку лишь в скалярном случае). 
Tеорема Е. Если $F=\left\{\gamma_{n}\right\} \subset \Gamma-$ множество Сидона, то с константой, зависящей только от константы Сидона $C(F)$,

$$
\left\|\sum_{n=1}^{m} a_{n} \gamma_{n}\right\|_{t} \asymp\left\|\sum_{n=1}^{m} a_{n} r_{n}\right\|_{t} \quad(t \geqslant 1) .
$$

Из теорем Е и 1 сразу получаем следующее утверждение, которое иным способом (но тоже с использованием теоремы Е) было доказано в [18].

Теорема 3. Всякая бесконечная система Сидона $F=\left\{\gamma_{n}\right\}_{n=1}^{\infty}$ характеров, определенных на компактной абелевой группе, әквивалентна по распределению системе Радемахера. Константа эквивалентности при этом зависит лишь от константы Сидона $C(F)$.

СлЕДСТВИЕ 3. Последовательности

$$
f_{n}(x)=\sin \left(2 \pi k_{n} x\right) \quad u \quad g_{n}(x)=\cos \left(2 \pi k_{n} x\right) \quad(x \in[0,1])
$$

при условии $k_{n+1} / k_{n} \geqslant \lambda>1$ әквивалентны по распределению системе Радемахера.

ЗАмечАниЕ 2. Отметим тот факт, что именно аналогия в поведении рядов Радемахера и лакунарных тригонометрических рядов (см. введение) послужила исходньм пунктом для выявления более глубоких связей между ними, приведя в результате к изучению систем, эквивалентных по распределению "модельной" системе Радемахера. Из работ, посвященных этой тематике, кроме [16]-[18] упомянем также заметку В.А. Родина и А. С. Белова [19]. В ней авторы, используя теоремy D, перенесли результаты работы [20] о поведении рядов Радемахера в симметричных пространствах функций на лакунарные тригонометрические ряды. Как сказано в этой заметке, в 1988 г. на Северо-Кавказской школе-конференции один из ее авторов докладьвал о совпадении с точностью до эквивалентности норм полиномов по системе Радемахера и лакунарных тригонометрических полиномов для многих симметричных пространств. Следствие 3 показывает, что последнее справедливо не для многих, а для всех симметричных пространств, так как норма функции в таком пространстве определяется распределением ее модуля [21; $§ 2.4]$. Это замечание позволяет, в частности, усилить некоторые результаты работы [19]. Так, например, в теореме 2 из нее, устанавливающей взаимно однозначные условия эквивалентности норм лакунарных тригонометрических полиномов в симметричном пространстве нормам последовательностей их коэффициентов в пространствах $l_{1}$ и $l_{2}$, требование интерполяционности симметричного пространства относительно пары $\left(L_{1}, L_{\infty}\right)$ является излишним.

\section{§ 3. Выделение подсистем, эквивалентных по распределению системе Радемахера}

ТЕОРемА 4. Пусть система с.в. $\left\{f_{n}\right\}_{n=1}^{\infty}$, определенных на вероятностном пространстве $(\Omega, \Sigma, \mathrm{P})$, содержит подпоследовательность $\left\{f_{n_{k}}\right\}_{k=1}^{\infty}$, удовлетворяющую условиям:

1) $\left|f_{n_{k}}(\omega)\right| \leqslant D(k=1,2, \ldots ; \omega \in \Omega)$;

2) $f_{n_{k}} \rightarrow 0$ слабо в $L_{2}$;

3) $d=\inf _{k=1,2, \ldots}\left\|f_{n_{k}}\right\|_{2}>0$. 
Тогда существует подсистема $\left\{\varphi_{i}\right\}_{i=1}^{\infty} \subset\left\{f_{n}\right\}_{n=1}^{\infty}$, для которой

$$
\left\|\sum_{i=1}^{\infty} a_{i} \varphi_{i}\right\|_{t} \asymp \mathscr{K}_{1,2}(\sqrt{t}, a) \quad\left(a=\left(a_{i}\right)_{i=1}^{\infty} \in l_{2}, \quad t \geqslant 1\right)
$$

с константой, зависящей только от $D$ u d.

ДокАЗАтЕльство. Как показано В.Ф. Гапошкиным (см. [2; теорема 1.3.2], подробное доказательство приведено в работе [1]), при условиях 1) и 2) можно выделить последовательность $\left\{g_{i}\right\} \subset\left\{f_{n_{k}}\right\}$, для которой при некотором $C_{1}=C_{1}(D)$ и всех $a=\left(a_{i}\right)_{i=1}^{\infty} \in l_{2}$ и $t \geqslant 1$ выполнено неравенство

$$
\left\|\sum_{i=1}^{\infty} a_{i} g_{i}\right\|_{t} \leqslant C_{1} \sqrt{t}\|a\|_{2}
$$

Тогда для произвольного разложения $\left(a_{i}\right)=\left(b_{i}\right)+\left(c_{i}\right),\left(b_{i}\right) \in l_{1},\left(c_{i}\right) \in l_{2}$,

$$
\begin{aligned}
\left\|\sum_{i=1}^{\infty} a_{i} g_{i}\right\|_{t} & \leqslant\left\|\sum_{i=1}^{\infty} b_{i} g_{i}\right\|_{\infty}+\left\|\sum_{i=1}^{\infty} c_{i} g_{i}\right\|_{t} \\
& \leqslant D \sum_{i=1}^{\infty}\left|b_{i}\right|+C_{1} \sqrt{t}\left(\sum_{i=1}^{\infty} c_{i}^{2}\right)^{1 / 2} \leqslant \max \left(C_{1} ; D\right)\left(\|b\|_{1}+\sqrt{t}\|c\|_{2}\right) .
\end{aligned}
$$

Отсюда по определению $\mathscr{K}$-функционала получаем

$$
\left\|\sum_{i=1}^{\infty} a_{i} g_{i}\right\|_{t} \leqslant \max \left(C_{1} ; D\right) \mathscr{K}_{1,2}(\sqrt{t}, a) \quad\left(a=\left(a_{i}\right)_{i=1}^{\infty} \in l_{2}, t \geqslant 1\right) .
$$

В доказательстве противоположного неравенства мы используем эквивалентность $\mathscr{K}$-функционала $\mathscr{K}_{1,2}(t, a)$ норме $Q\left(t^{2}\right)($ см. $(1.1)$ и $(1.2))$, а также оценку сверху $L_{q}$-норм $(q>1)$ модифицированных произведений Рисса (применение в аналогичных ситуациях классических произведений см. в $[2 ; \S 1.4]$ или $[10 ; \S 8.4])$.

Прежде всего, так как из условий 1) и 3$)$ теоремы следует: $0<d \leqslant\left\|g_{i}\right\|_{2} \leqslant D$ $(i=1,2, \ldots)$, то ввиду положительной однородности обеих частей соотношения (3.1) можно считать, что $\left\|g_{i}\right\|_{2}=1(i=1,2, \ldots)$.

Пусть числовая последовательность $\left(\varepsilon_{i}\right)_{i=1}^{\infty}$ такая, что

$$
\varepsilon_{i} \rightarrow 0, \quad 0<\varepsilon_{i}<\frac{1}{16} \min (1 ; D), \quad \sum_{k=i+1}^{\infty} \varepsilon_{k}<\varepsilon_{i} \quad(i=1,2, \ldots) .
$$

По условиям теоремы последовательность $g_{i}^{2}$ слабо компактна в $L_{2}$. Поэтому существует $\left\{h_{k}\right\} \subset\left\{g_{i}\right\}$ такая, что $h_{k}^{2} \rightarrow h$ слабо в этом пространстве, где $0 \leqslant h(\omega) \leqslant D^{2}$ и $\mathrm{E}(h)=1$. Вместе с $\left\{g_{i}\right\}$ последовательность $h_{k} \rightarrow 0$ слабо в $L_{2}$. Следовательно, можно найти номер $k_{1}$ так, что для функции $\varphi_{1}=h_{k_{1}}$ выполнено

$$
\left|\mathrm{E}\left(\varphi_{1}\right)\right|+\left|\mathrm{E}\left(h \varphi_{1}\right)\right|+\left|\mathrm{E}\left(\varphi_{1}^{2}-h\right)\right| \leqslant \frac{\varepsilon_{1}}{2 D} .
$$


Предположим, что номера $k_{1}<k_{2}<\cdots<k_{i-1}$ и функции $\varphi_{1}=h_{k_{1}}, \varphi_{2}=h_{k_{2}}$, $\ldots, \varphi_{i-1}=h_{k_{i-1}}(i \geqslant 2)$ уже выбраны. Найдем $k_{i}>k_{i-1}$ так, чтобы для $\varphi_{i}=h_{k_{i}}$ вьполнялось соотношение

$$
\begin{array}{r}
\sum\left\{\left|\mathrm{E}\left(\varphi_{j_{1}} \cdots \varphi_{j_{s}} \varphi_{i}\right)\right|+\left|\mathrm{E}\left(h \varphi_{j_{1}} \cdots \varphi_{j_{s}} \varphi_{i}\right)\right|+\left|\mathrm{E}\left[\varphi_{j_{1}} \cdots \varphi_{j_{s}}\left(\varphi_{i}^{2}-h\right)\right]\right|\right. \\
\left.+\sum_{l=1}^{s}\left|\mathrm{E}\left(\varphi_{j_{1}} \cdots \varphi_{j_{l-1}} \varphi_{j_{l}}^{2} \varphi_{j_{l+1}} \cdots \varphi_{j_{s}} \varphi_{i}\right)\right|\right\} \leqslant 2^{-i} D^{-1} \varepsilon_{i},
\end{array}
$$

где $\varphi_{j_{0}}=\varphi_{j_{s+1}}=1$, а сумма берется по всем наборам индексов $1 \leqslant j_{1}<j_{2}<$ $\cdots<j_{l}<\cdots<j_{s} \leqslant i-1(s=1,2, \ldots, i-1)$.

Покажем, что для последовательности $\left\{\varphi_{i}\right\}_{i=1}^{\infty}$ и произвольного $t \geqslant 1$ справедливо соотношение (3.1).

Пусть сначала $t \in \mathbb{N}$, а $\left\{A_{j}\right\}_{j=1}^{t}$ - произвольное разбиение натурального ряда на $t$ множеств. Для $N \in \mathbb{N}$ и $j=1,2, \ldots, t$ определим множества $A_{j}^{N}=\left\{i=1, \ldots, N: i \in A_{j}\right\}$ (возможно, некоторые из них пусты). Образуем произведения Рисса, состоящие из блоков, соответствуюших множествам $A_{j}^{N}$ :

$$
R_{N}(\omega)=\prod_{i=1}^{N}\left(1+b_{i} \varphi_{i}(\omega)\right)=\prod_{j=1}^{t} \prod_{i \in A_{j}^{N}}\left(1+b_{i} \varphi_{i}(\omega)\right)
$$

где $b_{i} \in \mathbb{R}$ удовлетворяют условиям

$$
\sum_{i \in A_{j}} b_{i}^{2} \leqslant D^{-2}
$$

Следуя классическому методу Сидона, для произвольной функции

$$
\varphi(\omega)=\sum_{i=1}^{\infty} a_{i} \varphi_{i}(\omega), \quad a=\left(a_{i}\right)_{i=1}^{\infty} \in l_{2},
$$

оценим снизу интеграл

$$
I_{N}=\int_{\Omega} R_{N}(\omega) \varphi(\omega) d \mathrm{P}(\omega)=\sum_{i=1}^{\infty} a_{i} \mathrm{E}\left(R_{N} \varphi_{i}\right)=\sum_{i=1}^{\infty} a_{i} \beta_{i, N}
$$

Здесь

$$
\begin{aligned}
& \beta_{i, N}=\mathrm{E}\left(R_{N} \varphi_{i}\right)=\int_{\Omega}\left[1+\sum_{k=1}^{N} b_{k} \varphi_{k}(\omega)+\sum_{1 \leqslant k_{1}<k_{2} \leqslant N} b_{k_{1}} b_{k_{2}} \varphi_{k_{1}}(\omega) \varphi_{k_{2}}(\omega)\right. \\
& \left.+\cdots+b_{1} b_{2} \cdots b_{N} \varphi_{1}(\omega) \cdots \varphi_{N}(\omega)\right] \varphi_{i}(\omega) d \mathrm{P}(\omega)=b_{i} \alpha_{i, N}+\gamma_{i, N}, \quad(3.8) \\
& \text { где } \alpha_{i, N}=1(i \leqslant N), \alpha_{i, N}=0(i>N), \mathrm{a} \\
& \gamma_{i, N}=\mathrm{E}\left(\varphi_{i}\right)+\sum_{k=1, k \neq i}^{N} b_{k} \mathrm{E}\left(\varphi_{k} \varphi_{i}\right)+\sum_{1 \leqslant k_{1}<k_{2} \leqslant N} b_{k_{1}} b_{k_{2}} \mathrm{E}\left(\varphi_{k_{1}} \varphi_{k_{2}} \varphi_{i}\right) \\
& +\cdots+\sum_{1 \leqslant k_{1}<\cdots<k_{s} \leqslant N} b_{k_{1}} \cdots b_{k_{s}} \mathrm{E}\left(\varphi_{k_{1}} \cdots \varphi_{k_{s}} \varphi_{i}\right)+\cdots+b_{1} \cdots b_{N} \mathrm{E}\left(\varphi_{1} \cdots \varphi_{N} \varphi_{i}\right) .
\end{aligned}
$$


Тогда

$$
\gamma_{i, N}=S_{1}^{i}+S_{2}^{i}+S_{3}^{i}
$$

где суммы $S_{k}^{i}(k=1,2,3)$ определяются следующим образом. Представляя

$$
\mathrm{E}\left(\varphi_{k_{1}} \cdots \varphi_{k_{s}} \varphi_{i}^{2}\right)=\mathrm{E}\left(\varphi_{k_{1}} \cdots \varphi_{k_{s}} h\right)+\mathrm{E}\left[\varphi_{k_{1}} \cdots \varphi_{k_{s}}\left(\varphi_{i}^{2}-h\right)\right],
$$

отнесем к $S_{1}^{i}$ все слагаемые, содержащие интегралы вида $\mathrm{E}\left(\varphi_{k_{1}} \cdots \varphi_{k_{s}} \varphi_{i}\right)$ или $\mathrm{E}\left[\varphi_{k_{1}} \cdots \varphi_{k_{s}}\left(\varphi_{i}^{2}-h\right)\right]$, где $k_{1}<k_{2}<\cdots<k_{s}<i$. В сумму $S_{2}^{i}$ включим все слагаемые с интегралами вида $\mathrm{E}\left(\varphi_{k_{1}} \cdots \varphi_{k_{l-1}} \varphi_{i} \varphi_{k_{l}} \cdots \varphi_{k_{s}}\right)$, где $k_{1}<k_{2}<\cdots<k_{l-1} \leqslant$ $i<k_{l}<\cdots<k_{s}, 1 \leqslant l \leqslant s$, а в $S_{3}^{i}$ - слагаемые с интегралами $\mathrm{E}\left(\varphi_{k_{1}} \cdots \varphi_{k_{s}} h\right)$.

Ввиду соотношений (3.5), (3.4) и (3.6) мы получим оценки

$$
\left|S_{1}^{i}\right| \leqslant \frac{\varepsilon_{i}}{D}, \quad\left|S_{2}^{i}\right| \leqslant \frac{1}{D} \sum_{k=i+1}^{\infty} \varepsilon_{k}<\frac{\varepsilon_{i}}{D} .
$$

Так как в каждое слагаемое суммы $S_{3}^{i}$ входит множитель $b_{i}$, то из (3.4) получаем

$$
\left|S_{3}^{i}\right| \leqslant \frac{\left|b_{i}\right|}{D} \sum_{i=1}^{\infty} \varepsilon_{i} \leqslant \frac{\left|b_{i}\right|}{8}
$$

(множитель $2^{-i}$ из (3.5) мы пока не используем). Кроме того, при $i>N$ суммы $S_{2}^{i}$ и $S_{3}^{i}$ в $(3.9)$ отсутствуют, и поэтому

$$
\sum_{i=N+1}^{\infty}\left|\gamma_{i, N}\right| \leqslant \frac{1}{D} \sum_{i=N+1}^{\infty} \varepsilon_{i} \leqslant \frac{1}{16 D}
$$

Пусть теперь $1 \leqslant i \leqslant N$. Для каждого $j=1,2, \ldots, t$ ввиду соотношений $(3.9)-(3.11),(3.4)$ и $(3.6)$

$$
\begin{aligned}
\left(\sum_{i \in A_{j}^{N}} \gamma_{i, N}^{2}\right)^{1 / 2} & \leqslant \sum_{k=1}^{3}\left(\sum_{i \in A_{j}^{N}}\left(S_{k}^{i}\right)^{2}\right)^{1 / 2} \\
& \leqslant \frac{2}{D} \sum_{i \in A_{j}^{N}} \varepsilon_{i}+\frac{1}{8}\left(\sum_{i \in A_{j}^{N}} b_{i}^{2}\right)^{1 / 2} \leqslant \frac{3}{8 D} .
\end{aligned}
$$

Соотношения (3.7) и (3.8) показывают, что

$$
\begin{aligned}
I_{N} & =\sum_{i=1}^{N} a_{i} b_{i}+\sum_{i=1}^{N} a_{i} \gamma_{i, N}+\sum_{i=N+1}^{\infty} a_{i} \gamma_{i, N} \\
& =\sum_{j=1}^{t}\left(\sum_{i \in A_{j}^{N}} a_{i} b_{i}\right)+\sum_{j=1}^{t}\left(\sum_{i \in A_{j}^{N}} a_{i} \gamma_{i, N}\right)+\sum_{i=N+1}^{\infty} a_{i} \gamma_{i, N} .
\end{aligned}
$$

Для каждого $j=1,2, \ldots, t$ выберем $b_{i}\left(i \in A_{j}^{N}\right)$ так, чтобы выполнялись условия (3.6) и

$$
\sum_{i \in A_{j}^{N}} a_{i} b_{i}=\frac{1}{D}\left(\sum_{i \in A_{j}^{N}} a_{i}^{2}\right)^{1 / 2},
$$


а также $N \in \mathbb{N}$ такое, что

$$
\|a\|_{2} \leqslant 2\left(\sum_{i=1}^{N} a_{i}^{2}\right)^{1 / 2} \leqslant 2 \sum_{j=1}^{t}\left(\sum_{i \in A_{j}^{N}} a_{i}^{2}\right)^{1 / 2}
$$

Тогда из предыдущего равенства и соотношений $(3.12),(3.13)$ и (3.4) получим

$$
\begin{aligned}
I_{N} & \geqslant \frac{1}{D} \sum_{j=1}^{t}\left(\sum_{i \in A_{j}^{N}} a_{i}^{2}\right)^{1 / 2}-\sum_{j=1}^{t}\left(\sum_{i \in A_{j}^{N}} a_{i}^{2}\right)^{1 / 2}\left(\sum_{i \in A_{j}^{N}} \gamma_{i, N}^{2}\right)^{1 / 2}-\sum_{i=N+1}^{\infty}\left|a_{i}\right|\left|\gamma_{i, N}\right| \\
& \geqslant \frac{5}{8 D} \sum_{j=1}^{t}\left(\sum_{i \in A_{j}^{N}} a_{i}^{2}\right)^{1 / 2}-\left(\sum_{i=N+1}^{\infty}\left|a_{i}\right|^{2}\right)^{1 / 2}\left(\sum_{i=N+1}^{\infty} \gamma_{i, N}^{2}\right)^{1 / 2} \\
& \geqslant \frac{1}{2 D} \sum_{j=1}^{t}\left(\sum_{i \in A_{j}^{N}} a_{i}^{2}\right)^{1 / 2} .
\end{aligned}
$$

Последнее неравенство показывает, что для каждого $t \in \mathbb{N}$ и произвольного разбиения $\left\{A_{j}\right\}_{j=1}^{t}$ натурального ряда найдутся достаточно большое $N$ и числа $b_{i}$ $(i=1,2, \ldots, N)$, удовлетворяющие условиям $(3.6)$, такие, что

$$
I_{N}=\int_{\Omega} R_{N}(\omega) \varphi(\omega) d \mathrm{P}(\omega) \geqslant \frac{1}{3 D} \sum_{j=1}^{t}\left(\sum_{i \in A_{j}} a_{i}^{2}\right)^{1 / 2} .
$$

Следующая часть доказательства - оценка интеграла $I_{N}$ сверху выражением $C\|\varphi\|_{t}$, где $C>0$ - некоторая константа. Ввиду неравенства Гёльдера

$$
\left|I_{N}\right| \leqslant\left\|R_{N}\right\|_{t^{\prime}}\|\varphi\|_{t}, \quad t^{\prime}=\frac{t}{t-1},
$$

и неотрицательности произведений Рисса для этого достаточно оценить сверху величину

$$
L_{N}=\left\|R_{N}\right\|_{t^{\prime}}^{t^{\prime}}=\int_{\Omega}\left[R_{N}(\omega)\right]^{t^{\prime}} d \mathrm{P}(\omega)
$$

(при доказательстве того, что $\left\{\varphi_{i}\right\}_{i=1}^{\infty}-$ система Сидона, требуется лиш оценка $L_{1}$-норм произведений Рисса [2; теорема 1.4.1]).

Пусть $t \in \mathbb{N}, t \geqslant 3$. По-прежнему $\left\{A_{j}\right\}_{j=1}^{t}-$ разбиение $\mathbb{N}, A_{j}^{N}=\{i=1, \ldots, N$ : $\left.i \in A_{j}\right\}$, числа $b_{i}$ удовлетворяют соотношениям (3.6).

Из элементарного неравенства $(1+x)^{y} \leqslant 1+y x(x \geqslant 1,0<y \leqslant 1)$ следует, что

$$
\begin{aligned}
{\left[R_{N}(\omega)\right]^{t^{\prime}} } & \leqslant \prod_{i=1}^{N}\left(1+b_{i} \varphi_{i}(\omega)\right)\left[1+(t-1)^{-1} b_{i} \varphi_{i}(\omega)\right] \\
& \leqslant \prod_{i=1}^{N}\left[1+\frac{D^{2}}{t-1} b_{i}^{2}+\frac{t}{t-1} b_{i} \varphi_{i}(\omega)\right] \\
& =\prod_{j=1}^{t} \prod_{i \in A_{j}^{N}}\left[1+\frac{D^{2}}{t-1} b_{i}^{2}+\frac{t}{t-1} b_{i} \varphi_{i}(\omega)\right]
\end{aligned}
$$


Пусть $m(B)$ - количество элементов множества $B \subset \mathbb{N}$. Тогда внутреннее произведение в последнем выражении будет равно сумме

$$
\begin{aligned}
& \left\{1+\frac{D^{2}}{t-1} \sum_{i \in A_{j}^{N}} b_{i}^{2}+\left(\frac{D^{2}}{t-1}\right)^{2} \sum_{\substack{i_{1}<i_{2} \\
i_{1}, i_{2} \in A_{j}^{N}}} b_{i_{1}}^{2} b_{i_{2}}^{2}+\cdots+\left(\frac{D^{2}}{t-1}\right)^{m\left(A_{j}^{N}\right)} \prod_{i \in A_{j}^{N}} b_{i}^{2}\right\} \\
& \quad+\sum_{C_{j} \subset A_{j}^{N}}\left[1+\left(\frac{D^{2}}{t-1}\right)^{m\left(A_{j}^{N}\right)-m\left(C_{j}\right)} \prod_{i \in A_{j}^{N} \backslash C_{j}} b_{i}^{2}\right]\left(\frac{t}{t-1}\right)^{m\left(C_{j}\right)} \prod_{i \in C_{j}} b_{i} \varphi_{i}(\omega),
\end{aligned}
$$

где последняя сумма берется по всем непустым подмножествам $C_{j}$ множества $A_{j}^{N}$. Ввиду неравенств (3.6) при $t \geqslant 3$ выражение в фигурных скобках не превосходит $(t-1) /(t-2)$. Поэтому из соотношений $(3.16),(3.17)$ и опять (3.6) следует:

$$
\begin{aligned}
L_{N} \leqslant & \left(\frac{t-1}{t-2}\right)^{t}+\left(\frac{t-1}{t-2}\right)^{t-1} \sum_{j=1}^{t} \sum_{C_{j} \subset A_{j}^{N}}\left(\frac{t}{t-1}\right)^{m\left(C_{j}\right)+1}\left|\mathrm{E}\left[\prod_{i \in C_{j}} \varphi_{i}(\omega)\right]\right| \\
& +\left(\frac{t-1}{t-2}\right)^{t-2} \sum_{1 \leqslant j_{1}<j_{2} \leqslant t} \sum_{C_{j_{1}} \subset A_{j_{1}}^{N}}\left(\frac{t}{C_{j_{2}} \subset A_{j_{2}}^{N}}\right)^{m\left(C_{j_{1}}\right)+m\left(C_{j_{2}}\right)+2} \\
& \times\left|\mathrm{E}\left[\prod_{k=1}^{2} \prod_{i \in C_{j_{k}}} \varphi_{i}(\omega)\right]\right| \\
& +\cdots+\left(\frac{t-1}{t-2}\right)^{t-s} \sum_{1 \leqslant j_{1}<\cdots<j_{s} \leqslant t} \sum_{C_{j_{1}} \subset A_{j_{1}}^{N}} \sum_{C_{j} \subset A_{j s}^{N}}\left(\frac{t}{t-1}\right)^{\sum_{k=1}^{s} m\left(C_{j_{k}}\right)+s} \\
& \times\left|\mathrm{E}\left[\prod_{k=1}^{s} \prod_{i \in C_{j_{k}}} \varphi_{i}(\omega)\right]\right| \\
+\cdots & +\sum_{C_{1} \subset A_{1}^{N}} \sum_{C_{t} \subset A_{t}^{N}}\left(\frac{t}{t-1}\right)^{\sum_{j=1}^{t} m\left(C_{j}\right)+t}\left|\mathrm{E}\left[\prod_{j=1}^{t} \prod_{i \in C_{j}} \varphi_{i}(\omega)\right]\right|
\end{aligned}
$$

Если $t \geqslant 3$, то

$$
\frac{t}{t-1} \leqslant 2 \text { и }\left(\frac{t-1}{t-2}\right)^{t-s}\left(\frac{t}{t-1}\right)^{s} \leqslant 4 e \quad(s=0,1, \ldots, t) .
$$

Кроме того, так как множества $A_{j}^{N}(j=1,2, \ldots, t)$ попарно не пересекаются, то под интегралами в последнем выражении стоят различные произведения попарно различных функций $\varphi_{i}(i=1,2, \ldots, N)$. При этом число их равно $m\left(C_{j}\right)$ $(j=1,2, \ldots, t)$ для слагаемых первой, $m\left(C_{j_{1}}\right)+m\left(C_{j_{2}}\right)\left(1 \leqslant j_{1}<j_{2} \leqslant t\right)$ - для слагаемых второй, $\ldots, \sum_{j=1}^{t} m\left(C_{j}\right)$ - для слагаемых последней суммы. Следовательно, максимальный из индексов функций $\varphi_{i}$ в каждом подынтегральном произведении не менњше, чем $m\left(C_{j}\right), m\left(C_{j_{1}}\right)+m\left(C_{j_{2}}\right), \ldots, \sum_{j=1}^{t} m\left(C_{j}\right)$ соответственно. В итоге из (3.18) и (3.19) получаем

$$
L_{N} \leqslant 4 e\left\{\sum_{i=1}^{N} 2^{i} \sum_{1 \leqslant j_{1}<\cdots<j_{s} \leqslant i-1}\left|\mathrm{E}\left(\varphi_{j_{1}} \cdots \varphi_{j_{s}} \varphi_{i}\right)\right|\right\},
$$


где внутренняя сумма берется по всем наборам индексов $1 \leqslant j_{1}<\cdots<j_{s} \leqslant i-1$. Поэтому из соотношений (3.4) и (3.5) следует, что

$$
L_{N} \leqslant \frac{4 e}{D} \sum_{i=1}^{\infty} \varepsilon_{i}<2
$$

откуда ввиду (3.15) и (3.16) для всех $N \in \mathbb{N}$

$$
\left|I_{N}\right| \leqslant 2\|\varphi\|_{t}
$$

Учитывая соотношение (3.14), из последнего неравенства получаем

$$
\sum_{j=1}^{t}\left(\sum_{i \in A_{j}} a_{i}^{2}\right)^{1 / 2} \leqslant 6 D\|\varphi\|_{t}
$$

Таким образом, по лемме 1 для натуральных $t \geqslant 3$ и произвольных $a=\left(a_{i}\right)_{i=1}^{\infty} \in l_{2}$ вьполнено неравенство

$$
\mathscr{K}_{1,2}(\sqrt{t}, a) \leqslant 6 \sqrt{2} D\|\varphi\|_{t} .
$$

Проверим его справедливость для $t=1,2$.

Покажем, что построенная нами система $\left\{\varphi_{i}\right\}_{i=1}^{\infty}-$ базисная последовательность Рисса, т.е.

$$
\left\|\sum_{i=1}^{\infty} a_{i} \varphi_{i}\right\|_{2} \asymp\left\|\left(a_{i}\right)\right\|_{2} .
$$

Действительно, так как мы считаем, что $\left\|\varphi_{i}\right\|_{2}=1$, то ввиду (3.5) для произвольного $m \in \mathbb{N}$

$$
\begin{aligned}
\left\|\sum_{i=1}^{m} a_{i} \varphi_{i}\right\|_{2}^{2} & =\sum_{i=1}^{m} a_{i}^{2}+2 \sum_{1 \leqslant i<j \leqslant m} a_{i} a_{j} \mathrm{E}\left(\varphi_{i} \varphi_{j}\right) \\
& \geqslant \sum_{i=1}^{m} a_{i}^{2}\left\{1-2\left[\sum_{1 \leqslant i<j \leqslant m}\left(\mathrm{E}\left(\varphi_{i} \varphi_{j}\right)\right)^{2}\right]^{1 / 2}\right\} \geqslant \frac{1}{2}\left\|\left(a_{i}\right)\right\|_{2}^{2} .
\end{aligned}
$$

Аналогично

$$
\left\|\sum_{i=1}^{m} a_{i} \varphi_{i}\right\|_{2}^{2} \leqslant \frac{3}{2}\left\|\left(a_{i}\right)\right\|_{2}^{2}
$$

и (3.22) доказано.

Соотношение (3.2) показывает, что $\left\{g_{i}\right\}_{i=1}^{\infty}$, а значит, и $\left\{\varphi_{i}\right\}_{i=1}^{\infty}-S_{p}$-система для любого $p<\infty$. Поэтому она является системой Банаха [2; следствие 1.3.1], т.е. с некоторым $M=M(D)$

$$
\|a\|_{2} \leqslant M\left\|\sum_{i=1}^{m} a_{i} \varphi_{i}\right\|_{1} \quad\left(a=\left(a_{i}\right)_{i=1}^{\infty} \in l_{2}\right) .
$$

Так как $\|a\|_{2} \leqslant\|a\|_{1}$, то $\mathscr{K}\left(1, a ; l_{1}, l_{2}\right)=\|a\|_{2}$. Следовательно, ввиду вогнутости $\mathscr{K}$-функционала из соотношений (3.23) и (3.24) получаем

$$
\mathscr{K}_{1,2}(\sqrt{2}, a) \leqslant \sqrt{2} \mathscr{K}_{1,2}(1, a) \leqslant 2\|\varphi\|_{2}
$$


и

$$
\mathscr{K}_{1,2}(1, a) \leqslant M\|\varphi\|_{1} .
$$

Для произвольного $t \geqslant 1$ найдем натуральное $t_{0}$ такое, что $t_{0} \leqslant t<t_{0}+1$. Тогда опять ввиду вогнутости $\mathscr{K}$-функционала, а также соотношений $(3.21),(3.25)$ и (3.26)

$$
\mathscr{K}_{1,2}(\sqrt{t}, a) \leqslant \sqrt{t / t_{0}} \mathscr{K}_{1,2}\left(\sqrt{t_{0}}, a\right) \leqslant \sqrt{2} \mathscr{K}_{1,2}\left(\sqrt{t_{0}}, a\right) \leqslant C\|\varphi\|_{t},
$$

где $C=\sqrt{2} \max (2 ; M ; 6 \sqrt{2} D)$ зависит только от $D$.

Так как соотношение (3.3) выполнено и для системы $\left\{\varphi_{i}\right\}_{i=1}^{\infty}$, то доказательство теоремы закончено.

Следуюшие два утверждения - непосредственные следствия теорем 1 и 4.

ТЕОРЕма 5. Для того чтобы из системы $\left\{f_{n}\right\}_{n=1}^{\infty}$ c.в., определенных на вероятностном пространстве $(\Omega, \Sigma, \mathrm{P})$, можсно было выделить подсистему $\left\{\varphi_{i}\right\}_{i=1}^{\infty}$, әквивалентную по распределению системе Радемахера на [0, 1], необходимо и достаточно существование подпоследовательности $\left\{f_{n_{k}}\right\} \subset\left\{f_{n}\right\}$, удовлетворяющей условиям:

1) $\left|f_{n_{k}}(\omega)\right| \leqslant D(k=1,2, \ldots ; \omega \in \Omega)$;

2) $f_{n_{k}} \rightarrow 0$ слабо в $L_{2}$;

3) $d=\inf _{k=1,2, \ldots}\left\|f_{n_{k}}\right\|_{2}>0$.

При этом константа әквивалентности зависит лищь от $D$ и $d$.

ДоказАТЕльство. По теореме 4 выделим подпоследовательность $\left\{\varphi_{i}\right\}_{i=1}^{\infty} \subset$ $\left\{f_{n}\right\}_{n=1}^{\infty}$, для которой выполнено (3.1). Но тогда по теореме $1\left\{\varphi_{i}\right\} \stackrel{P}{\sim}\left\{r_{n}\right\}$ и достаточность доказана.

Необходимость условий 1) и 3) следует из определения эквивалентности по распределению и того, что аналогичными свойствами обладает система Радемахера. Как отмечалось во введении, система Радемахера является $S_{p}$-системой для любого $p<\infty$. Следовательно, это же свойство имеет и система, эквивалентная ей по распределению. Тем самым необходимость условия 2) следует из теоремы А, сформулированной во введении.

ЗАмЕчАНИЕ 3 . В связи с теоремой 5 напомним о следующей проблеме, поставленной Г. Алексичем $[22 ; \S 3.2]$. Из любой ли полной ортогональной системы функций можно выделить бесконечную мультипликативную или даже строго мультипликативную подсистему? Утверждение теоремы 5 дает положительный ответ на близкий вопрос: из любой системы с.в., обладающей свойствами 1)-3), можно выделить подсистему, эквивалентную по распределению строго мультипликативной (и даже состоящей из независимых функций) системе Радемахера.

ТЕОРемА 6. Если $\left\{f_{n}\right\}_{n=1}^{\infty}$ - ортонормированная последовательность с.в., определенных на вероятностном пространстве $(\Omega, \Sigma, \mathrm{P}),\left|f_{n}(\omega)\right| \leqslant D$ $(n=1,2, \ldots ; \omega \in \Omega)$, то из нее можсно выделить подпоследовательность $\left\{\varphi_{i}\right\}_{i=1}^{\infty}$, әквивалентную по распределению системе Радемахера. Константа этой әквивалентности зависит только от $D$.

Ясно, что последовательность $\left\{\varphi_{i}\right\}_{i=1}^{\infty}$, удовлетворяюшая условию $(3.1)$, является системой Сидона (как и $S_{p}$-системой для всех $p<\infty$ с константой $K_{p} \asymp \sqrt{p}$ ). Как оказывается, ее “сидоновость" является в определенном смысле усиленной. 
СЛЕДСТВИЕ 4. Предположим, что из системы с.в. $\left\{f_{n}\right\}_{n=1}^{\infty}$ можсно выделить подпоследовательность $\left\{f_{n_{k}}\right\}$, удовлетворяющую условиям 1)-3) теоремь 5. Тогда $\left\{f_{n}\right\}_{n=1}^{\infty}$ содержит подсистему $\left\{\varphi_{i}\right\}_{i=1}^{\infty}$ такую, что для некоторых констант $\alpha_{1}, \alpha_{2}, \alpha_{3}>0$, зависящих лишь от $D$ u $d$, и для любого полинома

$$
T(\omega)=\sum_{i=1}^{m} a_{i} \varphi_{i}(\omega)
$$

существует множество $E=E(T) \subset \Omega, \mathrm{P}(E)>\alpha_{1} 2^{-m}$ такое, что

$$
|T(\omega)| \geqslant \alpha_{2}\|T\|_{\infty} \geqslant \alpha_{3} \sum_{i=1}^{m}\left|a_{i}\right| \quad(\omega \in E) .
$$

ДокАЗАтЕльство. Согласно теореме 5 существует подпоследовательность $\left\{\varphi_{i}\right\} \subset\left\{f_{n}\right\}$ такая, что при некотором $C>0$ и всех $z>0$

$$
C^{-1}|\{|\widetilde{T}(x)|>C z\}| \leqslant \mathrm{P}\{|T(\omega)|>z\} \leqslant C\left|\left\{|\widetilde{T}(x)|>C^{-1} z\right\}\right|,
$$

где $\widetilde{T}(x)=\sum_{i=1}^{m} a_{i} r_{i}(x)(x \in[0,1])$. В частности, отсюда получаем

$$
C^{-1}\|T\|_{\infty} \leqslant\|\widetilde{T}\|_{\infty} \leqslant C\|T\|_{\infty}
$$

Поэтому из определения функций Радемахера следует

$$
\begin{aligned}
\mathrm{P}\left\{|T(\omega)|>\left(2 C^{2}\right)^{-1}\|T\|_{\infty}\right\} & \geqslant \mathrm{P}\left\{|T(\omega)|>(2 C)^{-1}\|\widetilde{T}\|_{\infty}\right\} \\
& \geqslant C^{-1}\left|\left\{|\widetilde{T}(x)|>2^{-1}\|\widetilde{T}\|_{\infty}\right\}\right| \geqslant C^{-1} 2^{-m} .
\end{aligned}
$$

Положим $\alpha_{1}=C^{-1}, \alpha_{2}=\left(2 C^{2}\right)^{-1}$ и $E=E(T)=\left\{\omega \in \Omega:|T(\omega)|>\alpha_{2}\|T\|_{\infty}\right\}$. Тогда для $\omega \in E$

$$
|T(\omega)|>\alpha_{2}\|T\|_{\infty} \geqslant \alpha_{2} C^{-1}\|\widetilde{T}\|_{\infty}=\alpha_{3} \sum_{i=1}^{m}\left|a_{i}\right|,
$$

где $\alpha_{3}=\alpha_{2} C^{-1}$.

Как уже отмечалось во введении (см. теорему В'), при тех же условиях на последовательность функций, измеримых на [0, 1], что и в теореме В, из нее можно выделить подсистему, обладающую свойством Сидона-Зигмунда, а при дополнительном условии (0.3) - свойством Сидона-Зигмунда в узком смысле. Как показывают два следующих результата, справедливы и несколько более сильные утверждения.

ТЕОРема 7. Пусть из последовательности $\left\{f_{n}\right\}_{n=1}^{\infty}$ функиий, измеримых на $[0,1]$, можсно выделить подпоследовательность $\left\{f_{n_{k}}\right\}$, удовлетворяющую условиям 1)-3) теоремь 5. Тогда существует подсистема $\left\{\varphi_{i}\right\} \subset\left\{f_{n}\right\}$ со следующим свойством:

для некоторого множсества $E \subset[0,1],|E|>0$, и любого промежутка $I \subset[0,1],|I \cap E|>0$, найдется номер $k_{0}=k_{0}(I)$ такой, что последовательность $\left\{\varphi_{i} \chi_{I}\right\}_{i=k_{0}}^{\infty}\left(\chi_{I}(x)=1, x \in I, u \chi_{I}(x)=0, x \notin I\right)$ әквивалентна по распределению системе Радемахера на $[0,1]$. Константа этой эквивалентности зависит от $D, d$ и промежутка $I$. 
ДокАЗАТЕЛЬСтво. По теореме 1 достаточно найти подсистему $\left\{\varphi_{i}\right\} \subset\left\{f_{n}\right\}$ и множество $E \subset[0,1],|E|>0$, такие, что для всякого промежутка $I \subset[0,1]$, $|I \cap E|>0$, сушествует номер $k_{0}=k_{0}(I)$ такой, что

$$
\left\|\sum_{i=1}^{\infty} a_{i} \varphi_{k_{0}+i-1} \chi_{I}\right\|_{t} \asymp \mathscr{K}_{1,2}(\sqrt{t}, a) \quad\left(a=\left(a_{i}\right)_{i=1}^{\infty}, t \geqslant 1\right)
$$

(константа эквивалентности зависит от $D, d$ и $I$ ).

Рассуждения, приводящие к последнему соотношению, в основном аналогичны тем, что использовались в доказательстве теоремы 4. Сделаем лишп несколько замечаний.

Прежде всего, неравенство (3.2) остается, конечно, справедливым, если функции $g_{i}$ заменить на функции $g_{i} \chi_{I}(I-$ произвольный промежуток из $[0,1])$.

Далее, как и в доказательстве теоремы 1.4 .2 из [2], подпоследовательность $\left\{\varphi_{i}\right\} \subset\left\{g_{i}\right\}$ можно выделить так, что соотношения типа (3.5) выполняются для интегралов по любому промежутку $I \subset[0,1]$.

И наконец, по известной лемме Марцинкевича [2; лемма 1.2.5] можно считать, что для некоторого множества $E \subset[0,1],|E|>0$, при любом $F \subset E,|F|>0$, вьполнено

$$
\liminf _{i \rightarrow \infty} \int_{F} \varphi_{i}^{2}(x) d x>0
$$

После этого фактически повторяется доказательство теоремы 4 , только интегралы по $[0,1]$ нужно заменить интегралами по промежутку $I$ такому, что $|I \cap E|>0$.

ТЕОРема 8. Предположим, что из последовательности $\left\{f_{n}\right\}_{n=1}^{\infty}$ функиий, измеримых на $[0,1]$, можно выделить подпоследовательность $\left\{f_{n_{k}}\right\}$, удовлетворяющую условиям 1) и 2) теоремы 5 , а такжсе условию (0.3).

Тогда существует подсистема $\left\{\varphi_{i}\right\} \subset\left\{f_{n}\right\}$ такая, ито для всякого промежутка $I \subset[0,1],|I|>0$, найдется номер $k_{0}=k_{0}(I)$, при котором последовательность $\left\{\varphi_{i} \chi_{I}\right\}_{i=k_{0}}^{\infty}$ әквивалентна по распределению системе Радемахера на $[0,1]$.

Доказательство теоремы 8 совершенно аналогично доказательству предыдущей теоремы, так как ввиду условия (0.3) можно считать, что выделенная подсистема $\left\{\varphi_{i}\right\}_{i=1}^{\infty}$ удовлетворяет соотношению (3.27) для любого множества $F \subset[0,1]$ положительной меры.

\section{§4. Плотность подсистем, эквивалентных по распределению системе Радемахера}

Рассмотрим задачу выделения подсистем из конечных равномерно ограниченных ортонормированных наборов $\left\{f_{n}\right\}_{n=1}^{N}$ функций, определенных на отрезке [0, 1]. Мы покажем, что любой такой набор содержит подсистему $\left\{f_{n_{i}}\right\}_{i=1}^{s}$ "логарифмической плотности" (т.е. $s \geqslant C \log _{2} N$ ), эквивалентную по распределению системе первых $s$ функций Радемахера с константой, не зависяшей от $s$. Этот результат является усилением теоремы С из введения, доказанной Б. С. Кашиным [9].

ТЕОРема 9. Пусть $\left\{f_{n}\right\}_{n=1}^{N}$ - ортонормированный набор функций, определенных на $[0,1],\left|f_{n}(x)\right| \leqslant D(n=1,2, \ldots, N)$. Тогда существует набор $\left\{f_{n_{i}}\right\}_{i=1}^{s}\left(1 \leqslant n_{1}<n_{2}<\cdots<n_{s} \leqslant N\right)$, əде $s \geqslant \max \left\{\left[\frac{1}{6} \log _{2} N\right] ; 1\right\}$, такой, что 
для некоторого $C>0$, зависящего только от $D$, всех $a_{i} \in \mathbb{R}(i=1,2, \ldots, s) u$ $z>0$

$C^{-1}\left|\left\{\left|\sum_{i=1}^{s} a_{i} r_{i}(x)\right|>C z\right\}\right| \leqslant\left|\left\{\left|\sum_{i=1}^{s} a_{i} f_{n_{i}}(x)\right|>z\right\}\right| \leqslant C\left|\left\{\left|\sum_{i=1}^{s} a_{i} r_{i}(x)\right|>C^{-1} z\right\}\right|$.

Для доказательства нам понадобятся две леммы. Первая из них хорошо известна [10; лемма 8.4.1].

ЛЕмма 3. Из любого набора функций $\left\{f_{n}\right\}_{n=1}^{N}\left(\log _{2} N \geqslant 6\right)$, удовлетворяющего условиям теоремы 9 , можно выбрать функиии $\left\{f_{n_{i}}\right\}_{i=1}^{s}\left(1 \leqslant n_{1}<n_{2}<\right.$ $\left.\cdots<n_{s} \leqslant N\right)$, где $s \geqslant\left[\frac{1}{6} \log _{2} N\right]$, для которых выполнено соотношение

$$
\sum_{\theta \in \mathscr{A}_{s}}\left\{\mathrm{E}\left[\prod_{i=1}^{s}\left(\frac{f_{n_{i}}(x)}{D}\right)^{\theta_{i}}\right]\right\}^{2} \leqslant 10^{-s}
$$

где $\mathscr{A}_{s}-$ совокупность всевозможсньх наборов $\theta=\left(\theta_{i}\right)_{i=1}^{s}$, таких, что
a) $\theta_{i}=0,1$ или 2 ;
b) $\sum_{i: \theta_{i}=1} 1 \geqslant 1$
c) $\sum_{i: \theta_{i}=2} 1 \leqslant 1$

Вторая лемма позволяет при определенных условиях продолжить равномерно ограниченный набор функций, определенных на $[0,1]$, на более широкое множество так, чтобы он стал мультипликативным, оставшись при этом равномерно ограниченным. Аналогичные результаты о продолжении до ортогонального набора см. в $[10 ; \oint 7.1]$.

Лемма 4. Пусть $\left\{g_{i}\right\}_{i=1}^{s}-$ набор функиий на $[0,1],\left|g_{i}(x)\right| \leqslant D(i=1,2, \ldots, s ;$ $x \in[0,1]) u$

$$
\max _{\theta \in \mathscr{A}_{s}^{\prime}}\left|\mathrm{E}\left[\prod_{i=1}^{s}\left(\frac{g_{i}(x)}{D}\right)^{\theta_{i}}\right]\right|<2^{-s}
$$

где $\mathscr{A}_{s}^{\prime}$ - совокупность всех наборов $\theta=\left(\theta_{i}\right)_{i=1}^{s} \in \mathscr{A}_{s}$ таких, что $\theta_{i}=0$ или 1 .

Существует мультипликативньй набор $\left\{h_{i}\right\}_{i=1}^{s}$ функиий на отрезке $[0,2]$ такой, что $h_{i}(x) \chi_{[0,1]}(x)=g_{i}(x) u\left\|h_{i}\right\|_{L_{\infty}[0,2]} \leqslant D(i=1,2, \ldots, s)$.

ДокАЗАТЕЛЬство. Представим:

$$
[1,2)=\bigcup_{k=1}^{2^{s}} \Delta_{k}, \quad \Delta_{k}=\left[a_{k-1}, a_{k}\right), \quad a_{k}=1+k 2^{-s} \quad\left(k=1,2, \ldots, 2^{s}\right)
$$

Произвольным образом установим взаимно однозначное соответствие между множеством $\mathscr{A}_{s}^{\prime}$ и семейством промежутков $\left\{\Delta_{k}\right\}_{k=1}^{2^{s}-1}$. Определим функции $h_{i}$ на каждом промежутке $\Delta_{k}$ из этого семейства.

Пусть $\theta=\left(\theta_{i}\right)_{i=1}^{s}-$ соответствующий $\Delta_{k}$ элемент множества $\mathscr{A}_{s}^{\prime}$. Обозначим $\left\{i_{j}\right\}_{j=1}^{m}=\left\{i=1,2, \ldots, s: \theta_{i}=1\right\}, i_{1}<i_{2}<\cdots<i_{m}(m=1,2, \ldots, s)$.

Для $\alpha \in \Delta_{k}$ определим функцию

$$
u_{\alpha}(x)=\chi_{\left[a_{k-1}, \alpha\right)}(x)-\chi_{\left[\alpha, a_{k}\right]}(x) \quad\left(x \in \Delta_{k}\right) .
$$


Тог да величина

$$
v(\alpha)=\int_{\Delta_{k}} u_{\alpha}(x) d x=2 \alpha-a_{k-1}-a_{k} \quad\left(a_{k-1} \leqslant \alpha \leqslant a_{k}\right)
$$

принимает все значения из отрезка $\left[-2^{-s}, 2^{-s}\right]$. Так как по условию (4.2) для функций $\bar{g}_{i}(x)=g_{i}(x) / D$ вьполнено: $\left|\mathrm{E}\left(\bar{g}_{i_{1}} \bar{g}_{i_{2}} \cdots \bar{g}_{i_{m}}\right)\right|<2^{-s}$, то найдется $\alpha_{k} \in$ $\left(a_{k-1}, a_{k}\right)$ такое, что

$$
v\left(\alpha_{k}\right)=-\mathrm{E}\left(\bar{g}_{i_{1}} \bar{g}_{i_{2}} \cdots \bar{g}_{i_{m}}\right)
$$

Пусть сначала $m \geqslant 2$. Возьмем систему кусочно постоянных функций $d_{j}(x)$, $x \in\left[a_{k-1}, \alpha_{k}\right)(j=1,2, \ldots, m-1)$, со следующими свойствами:

$$
\begin{aligned}
& \text { 1) }\left|d_{j}(x)\right| \equiv 1 \\
& \text { 2) } \int_{a_{k-1}}^{\alpha_{k}} d_{j_{1}} d_{j_{2}} \cdots d_{j_{l}} d x=0 \quad\left(1 \leqslant j_{1}<j_{2}<\cdots<j_{l} \leqslant m-1\right)
\end{aligned}
$$

(такую систему можно построить, например, перенеся на промежуток $\left[a_{k-1}, \alpha_{k}\right) \mathrm{c}$ отрезка $[0,1]$ функции Радемахера). Через $c_{j}(x), x \in\left[\alpha_{k}, a_{k}\right](j=1,2, \ldots, m-1)$, обозначим аналогичную систему, но построенную на промежутке $\left[\alpha_{k}, a_{k}\right]$.

Тогда если $x \in \Delta_{k}$, то

$$
\begin{aligned}
h_{i}(x) & \equiv 0 \quad\left(i \neq i_{j}, j=1,2, \ldots, m\right), \\
h_{i_{j}}(x) & =D\left[d_{j}(x) \chi_{\left[a_{k-1}, \alpha_{k}\right)}(x)+c_{j}(x) \chi_{\left[\alpha_{k}, a_{k}\right]}(x)\right] \quad(j=1,2, \ldots, m-1)
\end{aligned}
$$

и

$$
h_{i_{m}}(x)=D\left[\prod_{j=1}^{m-1} d_{j}(x) \chi_{\left[a_{k-1}, \alpha_{k}\right)}(x)-\prod_{j=1}^{m-1} c_{j}(x) \chi_{\left[\alpha_{k}, a_{k}\right]}(x)\right]
$$

Ввиду соотношений (4.4) и (4.3)

$$
\begin{aligned}
\int_{\Delta_{k}} h_{i_{1}} h_{i_{2}} \cdots h_{i_{m}} d x & =D^{m} \int_{\Delta_{k}} u_{\alpha_{k}}(x) d x \\
& =D^{m} v\left(\alpha_{k}\right)=-\mathrm{E}\left(g_{i_{1}} g_{i_{2}} \cdots g_{i_{m}}\right) .
\end{aligned}
$$

В то же время для любого другого набора индексов $1 \leqslant i_{1}^{\prime}<\cdots<i_{k}^{\prime} \leqslant s$ из определения функций $h_{i}(i=1,2, \ldots, s)$, а также из (4.4) следует

$$
\int_{\Delta_{k}} h_{i_{1}^{\prime}} h_{i_{2}^{\prime}} \cdots h_{i_{l}^{\prime}} d x=0
$$

Если же $m=1$, то положим

$$
h_{i}(x) \equiv 0 \quad\left(i \neq i_{1}\right), \quad h_{i_{1}}(x)=D u_{\alpha_{k}}(x)
$$

и соотношения (4.5) и (4.6) опять выполнены.

Доопределим функции $h_{i}(x)$ на всем отрезке [0,2]: $h_{i}(x)=g_{i}(x)(x \in[0,1])$, $h_{i}(x)=0\left(x \in \Delta_{2^{s}}\right)$. Тогда ввиду $(4.5)$ и (4.6) набор $\left\{h_{i}\right\}_{i=1}^{s}$ - мультипликативный на отрезке $[0,2]$ и $\left|h_{i}(x)\right| \leqslant D$. 
ДоКАЗАТЕЛЬСТВо ТЕОРЕМЫ 9. Ввиду теоремы 1 и замечания 1 после нее достаточно показать, что существует набор $\left\{f_{n_{i}}\right\}_{i=1}^{s}, s \geqslant \max \left\{\left[\frac{1}{6} \log _{2} N\right] ; 1\right\}$, такой, что с некоторой константой, зависящей только от $D$,

$$
\left\|\sum_{i=1}^{s} a_{i} f_{n_{i}}\right\|_{t} \asymp \mathscr{K}_{1,2}(\sqrt{t}, a) \quad\left(t \geqslant 1, a=\left(a_{i}\right)_{i=1}^{s}\right),
$$

где по-прежнему $\mathscr{K}_{1,2}(\sqrt{t}, a)=\mathscr{K}\left(\sqrt{t}, a ; l_{1}, l_{2}\right)$.

С помощью леммы 3 выделим подсистему $\left\{f_{n_{i}}\right\}_{i=1}^{s}\left(s \geqslant \max \left\{\left[\frac{1}{6} \log _{2} N\right] ; 1\right\}\right)$ так, чтобы она удовлетворяла условию (4.1). Так как $\mathscr{A}_{s}^{\prime} \subset \mathscr{A}_{s}$, то одновременно для $g_{i}=f_{n_{i}}$ выполнено и соотношение (4.2). Поэтому по лемме 4 набор $\left\{f_{n_{i}}\right\}_{i=1}^{s}$ можно продолжить до мультипликативного набора $\left\{h_{i}\right\}_{i=1}^{s}$ на $[0,2],\left|h_{i}(x)\right| \leqslant D$. Но тогда функции $h_{i}^{\prime}(x)=h_{i}(2 x)$ образуют мультипликативную систему уже на $[0,1]$, $\left|h_{i}^{\prime}(x)\right| \leqslant D$, и поэтому по следствию 3 из [16] для произвольных $t \geqslant 1$ и $a=\left(a_{i}\right)_{i=1}^{s}$

$$
\left\|\sum_{i=1}^{s} a_{i} h_{i}^{\prime}\right\|_{L_{t}[0,1]} \leqslant C_{1} \sqrt{t}\|a\|_{2}
$$

где $C_{1}>0$ зависит лишь от $D$. Следовательно,

$$
\left\|\sum_{i=1}^{s} a_{i} f_{n_{i}}\right\|_{t} \leqslant 2 C_{1} \sqrt{t}\|a\|_{2}
$$

откуда точно так же, как при доказательстве теоремы 4 (см. неравенство (3.20)), получаем

$$
\left\|\sum_{i=1}^{s} a_{i} f_{n_{i}}\right\|_{t} \leqslant C \mathscr{K}_{1,2}(\sqrt{t}, a) \quad(t \geqslant 1),
$$

где $C=\max \left(D ; 2 C_{1}\right)$ зависит только от $D$.

Для доказательства противоположного неравенства обозначим

$$
a=\left(a_{i}\right)_{i=1}^{s} \quad \text { и } P(x)=\sum_{i=1}^{s} a_{i} f_{n_{i}}(x) .
$$

Пусть сначала $t \in \mathbb{N}, t \geqslant 3$. Тогда если $s \leqslant 16 D^{2}$, то по определению $\mathscr{K}$-функционала

$$
\|P\|_{t} \geqslant\|P\|_{2}=\|a\|_{2} \geqslant \frac{1}{\sqrt{s}} \sum_{i=1}^{s}\left|a_{i}\right| \geqslant \frac{1}{4 D} \mathscr{K}_{1,2}(\sqrt{t}, a) .
$$

Поэтому достаточно рассмотреть случай, когда

$$
s>16 D^{2} .
$$

Образуем произведения Рисса

$$
R_{s}(x)=\prod_{i=1}^{s}\left(1+\frac{b_{i}}{D} f_{n_{i}}(x)\right) .
$$


Для произвольного разбиения $\left\{A_{j}\right\}_{j=1}^{t}$ множества $\{1,2, \ldots, s\}$ на $t$ непересекающихся подмножеств (возможно, некоторые из них пусты) потребуем, чтобы

$$
\sum_{i \in A_{j}} b_{i}^{2} \leqslant 1 \quad(j=1,2, \ldots, t)
$$

Оценим снизу интеграл

$$
\begin{aligned}
I_{s} & =\int_{0}^{1} P(x) R_{s}(x) d x \\
& =\sum_{i=1}^{s} a_{i} \int_{0}^{1} f_{n_{i}}(x) d x+\sum_{i=1}^{s} a_{i} \int_{0}^{1} f_{n_{i}}(x) \sum_{\theta \in \mathscr{A}_{s}^{\prime}} \prod_{k=1}^{s}\left[\frac{b_{k}}{D} f_{n_{k}}(x)\right]^{\theta_{k}} d x .
\end{aligned}
$$

Перегруппировав слагаемые, получим

$$
I_{s}=D^{-1} \sum_{i=1}^{s} a_{i} b_{i}+\sum_{i=1}^{s} a_{i} \gamma_{i}
$$

где

$$
\begin{gathered}
\gamma_{i}=\int_{0}^{1} f_{n_{i}}(x) d x+\int_{0}^{1} f_{n_{i}}(x) \sum_{\theta \in \mathscr{A}_{s}^{\prime}(i)} \prod_{k=1}^{s}\left[\frac{b_{k}}{D} f_{n_{k}}(x)\right]^{\theta_{k}} d x, \\
\mathscr{A}_{s}^{\prime}(i)=\mathscr{A}_{s}^{\prime} \backslash\left\{\theta^{i}\right\}, \quad \theta^{i}=\left(\theta_{j}^{i}\right), \quad \theta_{i}^{i}=1, \quad \theta_{j}^{i}=0(j \neq i) .
\end{gathered}
$$

Ввиду условия (4.1) и того, что $\left|b_{i}\right| \leqslant 1(i=1,2, \ldots, s)$, после применения неравенства Коши-Буняковского получим

$$
\sum_{i=1}^{s}\left|\gamma_{i}\right| \leqslant D \sum_{\theta \in \mathscr{A}_{s}}\left|\int_{0}^{1} \prod_{k=1}^{s}\left[\frac{f_{n_{k}}(x)}{D}\right]^{\theta_{k}} d x\right| \leqslant D\left(s 2^{s}\right)^{1 / 2} 10^{-s / 2}=D\left(s 5^{-s}\right)^{1 / 2} .
$$

Так как из-за $(4.11)\left(s 5^{-s}\right)^{1 / 2} \leqslant 8 / s<2^{-1} D^{-2}$, то отсюда

$$
\sum_{i=1}^{s}\left|\gamma_{i}\right| \leqslant \frac{1}{2 D}
$$

Выберем для каждого $j=1,2, \ldots, t$ числа $b_{i}\left(i \in A_{j}\right)$ так, чтобы выполнялось (4.12) и

$$
\sum_{i \in A_{j}} a_{i} b_{i}=\left(\sum_{i \in A_{j}} a_{i}^{2}\right)^{1 / 2}
$$

Тогда из (4.13) и (4.14) получаем

$$
\begin{aligned}
I_{s} & \geqslant \frac{1}{D} \sum_{j=1}^{t}\left(\sum_{i \in A_{j}} a_{i}^{2}\right)^{1 / 2}-\sum_{j=1}^{t}\left|\sum_{i \in A_{j}} a_{i} \gamma_{i}\right| \\
& \geqslant \frac{1}{D} \sum_{j=1}^{t}\left(\sum_{i \in A_{j}} a_{i}^{2}\right)^{1 / 2}-\sum_{j=1}^{t}\left(\sum_{i \in A_{j}} a_{i}^{2}\right)^{1 / 2}\left(\sum_{i \in A_{j}}\left|\gamma_{i}\right|\right) \\
& \geqslant \frac{1}{2 D} \sum_{j=1}^{t}\left(\sum_{i \in A_{j}} a_{i}^{2}\right)^{1 / 2} .
\end{aligned}
$$


Так как разбиение $\left\{A_{j}\right\}_{j=1}^{t}$ произвольно, то в итоге по лемме 1

$$
I_{s} \geqslant \frac{1}{2 \sqrt{2} D} \mathscr{K}_{1,2}(\sqrt{t}, a) \quad(t \in \mathbb{N}, t \geqslant 3)
$$

Оценим теперь интеграл $I_{s}$ сверху. По неравенству Гёльдера

$$
\left|I_{s}\right| \leqslant\left\|R_{s}\right\|_{t^{\prime}}\|P\|_{t}, \quad t^{\prime}=\frac{t}{t-1} .
$$

Точно так же, как в доказательстве теоремы 4 , можно показать, что для $t \geqslant 3$

$$
\left\|R_{s}\right\|_{t^{\prime}} \leqslant 4 e 2^{s} \sum_{\theta \in \mathscr{A}_{s}^{\prime}}\left|\mathrm{E}\left\{\prod_{i=1}^{s}\left[\frac{f_{n_{i}}(x)}{D}\right]^{\theta_{i}}\right\}\right|
$$

откуда ввиду (4.1), вложения $\mathscr{A}_{s}^{\prime} \subset \mathscr{A}_{s}$ и неравенства Коши-Буняковского получаем: $\left\|R_{s}\right\|_{t^{\prime}} \leqslant 4 e$. Поэтому $\left|I_{s}\right| \leqslant 4 e\|P\|_{t}$ и из (4.15) следует

$$
\mathscr{K}_{1,2}(\sqrt{t}, a) \leqslant 8 \sqrt{2} e D\|P\|_{t} \quad(t \in \mathbb{N}, t \geqslant 3) .
$$

Аналогичные соотношения имеют место также для $t=2$ и $t=1$. Действительно, так как система $\left\{f_{n}\right\}_{n=1}^{N}$ ортонормирована и $\mathscr{K}_{1,2}(1, a)=\|a\|_{2}$, то

$$
\mathscr{K}_{1,2}(\sqrt{2}, a) \leqslant \sqrt{2} \mathscr{K}_{1,2}(1, a)=\sqrt{2}\|P\|_{2} .
$$

Если же $t=1$, то ввиду (4.8), как и в доказательстве теоремњ 4, можно применить [2; следствие 1.3.1] и, значит,

$$
\mathscr{K}_{1,2}(1, a)=\|a\|_{2} \leqslant M\|P\|_{1},
$$

где $M>0$ зависит только от $D$.

Наконец, переходя стандартным образом от натуральных (см. (4.16)-(4.18)) к произвольным $t \geqslant 1$, получаем

$$
\mathscr{K}_{1,2}(\sqrt{t}, a) \leqslant C\|P\|_{t},
$$

где константа $C=\sqrt{2} \max (M ; \sqrt{2} ; 8 \sqrt{2} e D)$ зависит лишь от $D$. Поэтому ввиду (4.9) эквивалентность (4.7) выполняется с константой, зависящей только от $D$, и доказательство закончено.

ЗАмЕчАНИЕ 4. Пример тригонометрической системы показывает, что теорема 9 (точно так же, как и теорема С из введения) по порядку неулучшаема. Действительно, как доказал С.Б. Стечкин [23], если последовательность $\left\{\sqrt{2} \cos 2 \pi n_{k} x\right\}_{k=1}^{\infty}(x \in[0,1])$ является системой Сидона, то

$$
\sum_{k: n_{k}<N} 1 \leqslant C \ln N \quad(N=2,3, \ldots) .
$$

ЗАмечаниЕ 5. Доказанные в этом параграфе теоремы о выделении как бесконечных, так и конечных подсистем, эквивалентных по распределению системе Радемахера, окончательны в том смысле, что дальнейшее “прореживание” не может улучшить распределений соответствующих полиномов. Как легко проверить, для любой подпоследовательности $\left\{r_{n_{i}}\right\}_{i=1}^{\infty}$ системы Радемахера и произвольных $m \in \mathbb{N}, a_{i} \in \mathbb{R}(i=1,2, \ldots, m)$ и $z>0$

$$
\left|\left\{\left|\sum_{i=1}^{m} a_{i} r_{n_{i}}(x)\right|>z\right\}\right|=\left|\left\{\left|\sum_{i=1}^{m} a_{i} r_{i}(x)\right|>z\right\}\right| .
$$




\section{§5. $\mathscr{K}$-замкнутая представимость банаховых пар}

Назовем банахову пару $\left(X_{0}, X_{1}\right) \mathscr{K}$-замкнуто представимой в банаховой паре $\left(Y_{0}, Y_{1}\right)$, если сушествует линейный оператор $T: X_{0}+X_{1} \rightarrow Y_{0}+Y_{1}$ такой, что

a) оператор $T$ ограниченно и инъективно действует из $X_{0}$ в $Y_{0}$ и из $X_{1}$ в $Y_{1}$;

b) с некоторой константой $C>0$, не зависящей от $x \in X_{0}+X_{1}$ и $t>0$,

$$
\mathscr{K}\left(t, T x ; Y_{0}, Y_{1}\right) \asymp \mathscr{K}\left(t, x ; X_{0}, X_{1}\right) .
$$

В [24] (см. также [25]) показано, что пара $\left(l_{1}, l_{2}\right) \mathscr{K}$-замкнуто представима в паре $\left(L_{\infty}, G\right)$ пространств функций, измеримых на $[0,1]$. Через $G$ здесь обозначается замыкание пространства $L_{\infty}$ в пространстве Орлича $L_{N}, N(u)=e^{u^{2}}-1$. При этом оператор $T$ можно определить следуюшим образом:

$$
\operatorname{Ta}(x)=\sum_{n=1}^{\infty} a_{n} r_{n}(x) \quad\left(a=\left(a_{n}\right)_{n=1}^{\infty} \in l_{2}\right) .
$$

Здесь, как и выше, $\left\{r_{n}\right\}_{n=1}^{\infty}-$ система Радемахера на $[0,1]$.

Предположим, что последовательность функций $\left\{f_{n}\right\}_{n=1}^{\infty}$, измеримых на $[0,1]$, удовлетворяет свойствам 1)-3) теоремы 5. Тогда из нее можно выделить подсистему $\left\{\varphi_{i}\right\}$, эквивалентную по распределению системе Радемахера. Так как в [24] все доказательства используют лиш оценки распределений модулей полиномов по системе Радемахера, то все результаты этой работы будут справедливы и для $\left\{\varphi_{i}\right\}$. В частности, оператор

$$
T_{\varphi} a(x)=\sum_{i=1}^{\infty} a_{i} \varphi_{i}(x) \quad\left(a=\left(a_{i}\right)_{i=1}^{\infty} \in l_{2}\right)
$$

также осуществляет $\mathscr{K}$-замкнутую представимость пары $\left(l_{1}, l_{2}\right)$ в паре $\left(L_{\infty}, G\right)$.

Докажем теперь одно отрицательное утверждение о $\mathscr{K}$-замкнутой представимости пар, тесно связанное с предыдущим примером.

ТЕОРема 10. Пара $\left(l_{1}, l_{2}\right)$ не является $\mathscr{K}$-замкнуто представимой в паре $\left(L_{\infty}, L_{2}\right)$ пространств с.в., определенных на вероятностном пространстве $(\Omega, \Sigma, \mathrm{P})$.

ДокАЗАТЕЛЬСтво. Предположим, что, напротив, существует линейный оператор $T: l_{2} \rightarrow L_{2}$, для которого

$$
\|T a\|_{\infty} \asymp\|a\|_{1} \quad\left(a \in l_{1}\right), \quad\|T a\|_{2} \asymp\|a\|_{2} \quad\left(a \in l_{2}\right)
$$

и при некотором $C_{1}>0$

$$
C_{1}^{-1} \mathscr{K}_{1,2}(t, a) \leqslant \mathscr{K}\left(t, T a ; L_{\infty}, L_{2}\right) \leqslant C_{1} \mathscr{K}_{1,2}(t, a) \quad\left(a \in l_{2}, t>0\right),
$$

где по-прежнему $\mathscr{K}_{1,2}(t, a)=\mathscr{K}\left(t, a ; l_{1}, l_{2}\right)$.

Обозначим $f_{n}=T e_{n}$, где $e_{n}=\left(\delta_{n}^{j}\right), \delta_{n}^{n}=1, \delta_{n}^{j}=0(j \neq n)$. Тогда из $(5.1)$ получаем, что для некоторых $D>0$ и $d>0$

$$
D^{-1}\|a\|_{1} \leqslant\left\|\sum_{n=1}^{\infty} a_{n} f_{n}\right\|_{\infty} \leqslant D\|a\|_{1} \quad\left(a \in l_{1}\right)
$$


И

$$
d\|a\|_{2} \leqslant\left\|\sum_{n=1}^{\infty} a_{n} f_{n}\right\|_{2} \leqslant d^{-1}\|a\|_{2} \quad\left(a \in l_{2}\right)
$$

Отсюда, в частности,

$$
\left|f_{n}(\omega)\right| \leqslant D \quad(n=1,2, \ldots ; \omega \in \Omega) \text { и }\left\|f_{n}\right\|_{2} \geqslant d .
$$

Кроме того, из (5.3) следует, что $f_{n} \neq 0(n=1,2, \ldots)$ и для любых $k, m \in \mathbb{N}$, $k \leqslant m$,

$$
\left\|\sum_{n=1}^{k} a_{n} f_{n}\right\|_{2} \leqslant d^{-2}\left\|\sum_{n=1}^{m} a_{n} f_{n}\right\|_{2}
$$

Следовательно, система $\left\{f_{n}\right\}_{n=1}^{\infty}$ является базисом в замкнутой по норме $L_{2}$ линейной оболочке $Z$ этой системы [26; part I, $\S 1]$. Иначе говоря, $\left\{f_{n}\right\}_{n=1}^{\infty}-$ базисная последовательность Рисса, откуда для $g \in Z \mathrm{E}\left(g f_{n}\right) \rightarrow 0$ при $n \rightarrow \infty[2 ; \S 1.1]$. Если $g \in L_{2}$ произвольна, то $g=g_{1}+g_{2}, g_{1} \in Z, g_{2} \in Z^{\perp}\left(Z^{\perp}\right.$ - ортогональное дополнение к $Z)$ и $\mathrm{E}\left(g f_{n}\right)=\mathrm{E}\left(g_{1} f_{n}\right) \rightarrow 0$ при $n \rightarrow \infty$. Таким образом,

$$
f_{n} \rightarrow 0 \text { слабо в } L_{2} \quad(n \rightarrow \infty) .
$$

Соотношения (5.4) и (5.5) показывают, что для системы $\left\{f_{n}\right\}_{n=1}^{\infty}$ выполнены условия 1)-3) теоремы 5. Значит, сушествует подпоследовательность $\left\{\varphi_{i}\right\} \subset\left\{f_{n}\right\}$, эквивалентная по распределению системе Радемахера на $[0,1]$. Отсюда и из неравенства Хинчина (см. [3] или [6; § 4.5]) получаем:

$$
C_{2}^{-1}\|a\|_{2} \leqslant\left\|\sum_{i=1}^{\infty} a_{i} \varphi_{i}\right\|_{p} \leqslant C_{2}\|a\|_{2} \quad\left(a=\left(a_{i}\right)_{i=1}^{\infty} \in l_{2}\right)
$$

где константа $C_{2}>0$ зависит лишш от $D, d$ и $p \in[1, \infty)$.

В то же время из (5.2) следует

$$
C_{1}^{-1} \mathscr{K}_{1,2}(t, a) \leqslant \mathscr{K}\left(t, \sum_{i=1}^{\infty} a_{i} \varphi_{i} ; L_{\infty}, L_{2}\right) \leqslant C_{1} \mathscr{K}_{1,2}(t, a) \quad\left(a \in l_{2}, t>0\right) .
$$

Поэтому, применяя к парам $\left(L_{\infty}, L_{2}\right)$ и $\left(l_{1}, l_{2}\right)$ интерполяционньй функтор $(\cdot, \cdot)_{\theta, p}$ с параметрами $0<\theta<1, p=2 / \theta$ (см. $\S 1$ ), ввиду [11; теорема 5.2.1] получим, что

$$
\left\|\sum_{i=1}^{\infty} a_{i} \varphi_{i}\right\|_{p} \asymp\|a\|_{r, p}=\left\{\sum_{i=1}^{\infty}\left(a_{i}^{*}\right)^{p} i^{p / r-1}\right\}^{1 / p}
$$

где $r=2 /(2-\theta)<2$ (константа последней эквивалентности зависит только от $C_{1}$ и $\theta$ ). Так как $\|a\|_{r, p} \nsucc\|a\|_{2}$, то последнее соотношение противоречит неравенствам (5.6) при $p=2 / \theta$.

Автор выражает благодарность Б. С. Кашину за постановку задачи, решение которой составляет содержание $§ 4$. 


\section{Список литературы}

1. Асташкин С. В. О выделении подсистем, "мажорируемых" системой Радемахера // Матем. заметки. 1999. Т. 65. № 4. С. 483-495.

2. Гапошкин В. Ф. Лакунарные ряды и независимые функции // УМН. 1966. Т. 21. №6. C. 3-82.

3. Khintchine A. Über dyadische Brüche // Math. Z. 1923. V. 18. P. 109-116.

4. Zygmund A. Sur les séries trigonométric lacunaires // J. Lond. Math. Soc. (2). 1930. V. 5. № 2. P. 138-145.

5. Banach S. Sur les séries lacunaires // Bull. Int. Acad. Polon. Sci. A. 1933. № 4/8. P. 149-154.

6. Качмаж C., Штейнгауз Г. Теория ортогональных рядов. М.: Физматгиз, 1958.

7. Zygmund A. On lacunary trigonometric series // Trans. Amer. Math. Soc. 1932. V. 34. №3. P. 435-446.

8. Kaczmarz S., Steinhaus G. Le systéme orthogonal de M. Rademacher // Studia Math. 1930. V. 2. P. 231-247.

9. Кашин Б. С. О некоторых свойствах пространства тригонометрических полиномов с равномерной нормой // Труды МИАН. 1980. Т. 145. С. 111-116.

10. Кашин Б. С., Саакян А. А. Ортогоналшные ряды. М.: Наука, 1984.

11. Берг Й., Лефстрем Й. Интерполяционные пространства. Введение. М.: Мир, 1980.

12. Montgomery-Smith S. J. The distribution of Rademacher sums // Proc. Amer. Math. Soc. 1990. V. 109. № 2. P. 517-522.

13. Holmstedt T. Interpolation of quasi-normed spaces // Math. Scand. 1970. V. 26. P. 177-199.

14. Gluskin E. D., Kwapien S. Tail and moment estimates for sums of independent random variables with logarithmically concave tails // Studia Math. 1995. V. 114. № 3. P. 303-309.

15. Кахан ЖК.-П. Случайные функциональные ряды. М.: Мир, 1973.

16. Jakubowski J., Kwapien S. On multiplicative systems of functions // Bull. Acad. Pol. Sci. Ser. Sci. Math. 1979. V. 27. №9. P. 689-694.

17. Pisier G. Les inégalités de Khintchin-Kahane d'aprés C. Borell // Semin. Geom. des Espaces de Banach, Ec. politech., Cent. Math., 1977-1978. Expose № 7, 1978. P. 1-14.

18. Asmar N. H., Montgomery-Smith S. On the distribution of Sidon series // Ark. Mat. 1993. V. 31. № 1. P. 13-26.

19. Белов A. C., Родин B. A. Нормы лакунарных полиномов в функциональных пространствах // Матем. заметки. 1992. Т. 51. № 3. С. 137-139.

20. Rodin V.A., Semyonov E.M. Rademacher series in symmetric spaces // Anal. Math. 1975. V. 1. № 3. P. 207-222.

21. Крейн С.Г., Петунин Ю.И., Семенов Е.М. Интерполяция линейных операторов. М.: Наука, 1978.

22. Алексич Г. Проблемы сходимости ортогональных рядов. М.: ИЛ, 1963.

23. Стечкин С. Б. Об абсолютной сходимости рядов Фурье // Изв. АН СССР. Сер. матем. 1956. T. 20. C. $385-412$.

24. Асташкин C. В. Об интерполяции подпространств симметричных пространств, порожденных системой Радемахера // Изв. РАЕН. Сер. МММИУ. 1997. Т. 1. № 1. С. 18-35.

25. Асташкин C. В. О рядах по системе Радемахера, "близких" к $L_{\infty} / /$ Функц. анализ и его прилож. 1998. Т. 32. № 3. С. 62-65.

26. Lindenstrauss J., Tzafriri L. Classical Banach spaces. Berlin: Springer-Verlag, 1977.

Самарский государственный университет

E-mail: astashkn@ssu.samara.ru 